\title{
Closed Maximality Principles: Implications, Separations and Combinations*
}

\author{
Gunter Fuchs \\ Institut für Mathematische Logik und Grundlagenforschung \\ Westfälische Wilhelms-Universität Münster \\ Einsteinstr. 62 \\ 48149 Münster, Germany \\ e-mail: gfuchs@math.uni-muenster.de
}

March 7, 2008

\begin{abstract}
I investigate versions of the Maximality Principles for the classes of forcings which are $<\kappa$-closed, $<\kappa$-directed-closed, or of the form $\operatorname{Col}(\kappa,<$ $\lambda)$. These principles come in many variants, depending on the parameters which are allowed. I shall write $\operatorname{MP}_{\Gamma}(A)$ for the maximality principle for forcings in $\Gamma$, with parameters from $A$. The main results of this paper are:

- The principles have many consequences, such as $<\kappa$-closed-generic $\Sigma_{2}^{1}\left(H_{\kappa}\right)$ absoluteness, and imply, e.g., that $\nabla_{\kappa}$ holds. I give an application to the automorphism tower problem, showing that there are Souslin trees which are able to realize any equivalence relation, and hence that there are groups whose automorphism tower is highly sensitive to forcing.

- The principles can be separated into a hierarchy which is strict, for many $\kappa$.

- Some of the principles can be combined, in the sense that they can hold at many different $\kappa$ simultaneously.

The possibilities of combining the principles are limited, though: While it is consistent that $\mathrm{MP}_{<\kappa-\operatorname{closed}}\left(H_{\kappa^{+}}\right)$holds at all regular $\kappa$ below any fixed $\alpha$, the "global" maximality principle, stating that $\mathrm{MP}_{<\kappa-\text { closed }}\left(H_{\kappa} \cup\right.$ $\{\kappa\})$ holds at every regular $\kappa$, is inconsistent. In contrast to this, it is equiconsistent with ZFC that the maximality principle for directed-closed forcings without any parameters holds at every regular cardinal. It is also
\end{abstract}

\footnotetext{
* This article was published in the Journal of Symbolic Logic, issue 73, vol. 01, pp. 276-308, 2008. The copyright is owned by the Association for Symbolic Logic.

Mathematical Subject Classification 2000: 03E35, 03E40, 03E45, $03 E 55$.

Keywords: Forcing Axioms, Maximality Principles
} 
consistent that every local statement with parameters from $H_{\kappa}+$ that's provably $<\kappa$-closed-forceably necessary is true, for all regular $\kappa$.

\section{Introduction}

The maximality principle MP is the following appealing axiom scheme: Every sentence that can be forced to be true in such a way that it stays true in every further forcing extension, is already true. The principle was introduced in [SV01] by Jonathan Stavi and Jouko Väänänen, using a slightly different formulation, and focussing mainly on cardinal preserving forcing notions. Inspired by an idea of Christophe Chalons (see [Cha00]), it was then rediscovered by Joel Hamkins and analyzed in a more general context in [Ham03b].

Hamkins emphasizes the connection to modal logic, and since it allows to express the principle, and variations of it, very elegantly, I shall adopt modal terminology as well. Namely, given a class $\Gamma$ of notions of forcing, let's say that a statement is $\Gamma$-possible, or $\Gamma$-forceable, if it is true in a forcing extension by a forcing notion from $\Gamma$. It is $\Gamma$-necessary (or $\Gamma$-persistent, to use terminology from [SV01]) if it holds in $\mathrm{V}$ and in any forcing extension by a forcing notion from $\Gamma$. The principle $\mathrm{MP}_{\Gamma}$ now says that every sentence $\varphi$ which is $\Gamma$-forceably $\Gamma$-necessary (i.e., the sentence " $\varphi$ is $\Gamma$-necessary" is $\Gamma$-forceable) is true. So in the original principle MP, $\Gamma$ is the class of all forcing notions.

To emphasize: In this setup, $\Gamma$ is a class of forcing notions that is defined by a formula $\varphi_{\Gamma}(x)$ (maybe containing parameters). In compound modal expressions, this formula is reinterpreted in forcing extensions. So, for example $\diamond_{\Gamma} \square_{\Gamma} \psi$ means that there is a notion of forcing $\mathbb{P}$ with $\varphi_{\Gamma}(\mathbb{P})$ such that $\mathbb{P}$ forces "for all $\mathbb{Q}$ with $\varphi_{\Gamma}(\mathbb{Q}), \mathbb{Q}$ forces $\psi$ ". In all the cases I am going to consider, there are obvious and natural formulae defining the particular classes of forcings.

There are many ways to modify the principle, for example by allowing certain classes of parameters, by restricting the class $\Gamma$, for example to ccc forcings, by demanding that the principle (with an allowed class of parameters) is necessary (with the class of parameters reinterpreted in the forcing extensions), et cetera. Many possibilities have been analyzed by George Leibman in [Lei04], and a few of the results presented in the present work overlap with research done independently by Leibman. I shall refer to his work whenever this occurs.

Here, I shall focus on the case when $\Gamma$ is a subclass of the class of $<\kappa$-closed notions of forcing, where $\kappa$ is a regular cardinal. The cases which I am interested in are those where $\Gamma$ consists of all $<\kappa$-closed forcings, or only of those that are $<\kappa$-directed-closed, or only of those of the form $\operatorname{Col}(\kappa, \lambda)$ or $\operatorname{Col}(\kappa,<\lambda)$, that is, the class of collapses to $\kappa$ (see Definition 2.1). I refer to these principles as the closed maximality principles at $\kappa$.

The following are the main aspects under which I investigate these principles.

Firstly, their consistency strength is of interest. This is the natural starting point when analyzing a new axiom, and at the same time, it is the aspect which bears the least surprises. This is because one may view the original maximality principle as the special case of a closed maximality principle at $\kappa=\omega$, and 
indeed, the results from [Ham03b] carry over in pretty much the expected way. It is also this aspect in which there are some overlaps with [Lei04]. In addition, I investigate questions concerning the compatibility of the principles with $\kappa$ being a large cardinal, and the consistency strength of the closed maximality principles at some $\kappa$ which satisfies a large cardinal axiom. Results in that area are to be found in sections 2 and 3 .

Secondly, and in part overlapping with the first aspect, I was particularly interested in outright implications of the maximality principles. There is a surprising variety of consequences, many of them concerning the existence of $\kappa$-trees with special properties, and generic $\Sigma_{2}^{1}\left(H_{\kappa}\right)$ absoluteness for $<\kappa$-closed forcing. For example, the combinatorial principle $\diamond_{\kappa}$ follows. A more elaborate example is the existence of $\kappa^{+}$-sequences of $\kappa^{+}$-Souslin trees which are able to realize any equivalence relation on $\kappa^{+}$, which shows that there are groups whose automorphism towers are highly sensitive to forcing extensions. I find the consequences of the closed maximality principles very interesting, because they are so unusual for forcing axioms, which mostly imply that $\mathrm{CH}$ fails. I organized things in such a way that results on the consistency strength of the principles are deferred until they become corollaries of results on consequences of the principles. One of these is that the necessary form of the maximality principle for $<\kappa$-closed forcings at uncountable $\kappa$ is inconsistent. Results in these directions are in section 3 .

Thirdly, I was interested in the relationships between the various maximality principles I introduced: Which implications hold between the principles, which of them can be reversed, et cetera. Results are strewn into the paper when they fit in; they can be found in sections 2.1,3 and 4 . Fixing $\kappa$, the closed maximality principle for $\operatorname{Col}(\kappa)$ implies that for $<\kappa$-directed-closed forcings, which in turn implies the principle for $<\kappa$-closed forcings. It turns out that the principles can be separated for many $\kappa$, in the sense that these implications cannot be reversed.

I also observed a fascinating phenomenon, namely that some of the closed principles can be combined, in the sense that they hold at several regular cardinals simultaneously. I analyze in section 5 how to obtain models in which the maximality principles for directed closed forcings hold at many regular cardinals, allowing parameters. I also produce models where the global "very lightface" maximality principle holds, i.e., the principle stating that the maximality principle for directed closed forcings holds at every regular cardinal simultaneously, albeit without parameters. Finally, I construct models in which all "local" consequences (in a sense that's made precise) of the global boldface principle hold.

Lastly, in section 6 , I provide a principal limitation of the extent to which versions of the maximality principles for closed forcings with parameters can be combined. In particular, the global principle stating that the maximality principle for $<\kappa$-closed forcings, with parameters from $H_{\kappa} \cup\{\kappa\}$, holds at every regular cardinal simultaneously, is inconsistent with ZFC. This is interesting, because the combination of these principles through the first $\alpha$ regular cardinals is no stronger than ZFC. It is also in contrast with the corresponding global combination of the "very lightface" principles, which has no consistency strength, either. Moreover, I show that the boldface maximality principles for forcings 
in $\operatorname{Col}(\kappa)$ cannot hold simultaneously at more than two regular $\kappa$. Probably, refinements of my arguments can be used to show that they cannot be combined at all. This reveals a significant difference between the maximality principles for $<\kappa$-closed or $<\kappa$-directed-closed forcings on the one hand, and for forcings in $\operatorname{Col}(\kappa)$ on the other.

I would like to thank Joel Hamkins for introducing me to the subject of maximality principles. Thanks are also due to the referee for many helpful suggestions and remarks.

\section{Local maximality principles}

I would like to introduce the maximality principles for closed forcings in a formal and precise way now. In general, a parameter is needed to express them, which leads to the following

Definition 2.1 Given a list $\dot{c}_{1}, \ldots, \dot{c}_{n}$ of constant symbols, let $\mathcal{L}_{\dot{c}_{1}, \ldots, \dot{c}_{n}}$ be the language of set theory, augmented by these additional constant symbols.

If $S$ is some $\mathcal{L}_{\dot{\kappa}}$-term (of the form $\{x \mid \psi(x)\}$ ), then $\mathrm{MP}_{<\dot{\kappa} \text {-closed }}(S)$ is the scheme of formulae in $\mathcal{L}_{\dot{\kappa}}$ expressing that $\dot{\kappa}$ is a regular cardinal and that every $\mathcal{L}$-sentence with parameters from $S$ which is $<\dot{\kappa}$-closed-forceably necessary is true. The principle $\mathrm{MP}_{<\dot{\kappa}-\text { dir. cl. }}(S)$ is defined in the analogous manner.

Now let $\lambda$ be an ordinal and $\kappa$ a regular cardinal. Then $\operatorname{Col}(\kappa, \lambda)$ is the partial order consisting of partial functions $f: \kappa \longrightarrow \lambda$ of size less than $\kappa$, ordered by reverse inclusion. So in case $\lambda \geq \kappa^{+}$, this is the usual forcing to collapse $\lambda$ to $\kappa$. I allow for $\lambda$ to be less than $\kappa^{+}$, though, in which case, unless $\lambda=0$, subsets are added to $\kappa$, without collapsing cardinals. The case $\lambda=0$ is also allowed. Then $\operatorname{Col}(\kappa, \lambda)$ is trivial forcing. Further, $\operatorname{Col}(\kappa,<\lambda)$ is the forcing which collapses everything below $\lambda$ to $\kappa$. So, the partial order consists of partial functions $f: \kappa \times \lambda \longrightarrow \lambda$ of size less than $\kappa$, so that $f(\alpha, \beta)<\beta$. In other words, $f(\cdot, \beta) \in \operatorname{Col}(\kappa, \beta)$. The order is again reverse inclusion, so that $\operatorname{Col}(\kappa, \lambda)$ is isomorphic to the product $\prod_{\beta<\lambda} \operatorname{Col}(\kappa, \beta)$, with $<\kappa$-support.

Let

$$
\operatorname{Col}(\kappa)=\{\operatorname{Col}(\kappa,<\delta) \mid \delta \in \operatorname{On}\} \cup\{\operatorname{Col}(\kappa, \delta) \mid \delta \in \mathrm{On}\},
$$

and denote by $\operatorname{MP}_{\mathrm{Col}(\dot{\kappa})}(S)$ the scheme of formulae asserting that every $\mathcal{L}$ sentence with parameters from $S$ that is $\operatorname{Col}(\kappa)$-forceably necessary is already true.

I refer to these principles as the closed maximality principles at $\kappa$. Most of the time, I shall be interested in the case where $S$ is one of the following: $H_{\dot{\kappa}} \cup\{\dot{\kappa}\}, H_{\dot{\kappa}^{+}}$or $\emptyset$. Setting $S=H_{\dot{\kappa}^{+}}$yields what I call the boldface versions of the principles, $S=H_{\dot{\kappa}} \cup\{\dot{\kappa}\}$ yields the lightface versions, and $S=\emptyset$ gives the very lightface versions of the principles. ${ }^{1}$ If $\langle M, \kappa\rangle$ is a model of $\mathrm{MP}_{\langle\dot{\kappa} \text {-closed }}\left(H_{\dot{\kappa}} \cup\right.$

\footnotetext{
${ }^{1}$ It will turn out that the versions of the principles with parameter set $\{\dot{\kappa}\}$ imply their counterparts with parameter set $H_{\dot{\kappa}} \cup\{\dot{\kappa}\}$ - see Theorem 2.6 - and hence these are equivalent. This justifies referring to them as lightface principles, since it seems very natural to allow $\dot{\kappa}$ as a parameter, considering that $\dot{\kappa}$ is needed even to define the class of forcings.
} 
$\{\dot{\kappa}\})(S)$, I shall say that $M$ is a model of $\mathrm{MP}_{<\kappa-\operatorname{closed}}\left(H_{\kappa} \cup\{\kappa\}\right)$.

The principles $\mathrm{MP}_{<\kappa-\text { closed }}\left(H_{\kappa} \cup\{\kappa\}\right), \mathrm{MP}_{<\kappa-\text { closed }}\left(H_{\kappa^{+}}\right), \mathrm{MP}_{<\kappa-\text { dir. cl. }}\left(H_{\kappa} \cup\right.$ $\{\kappa\}), \mathrm{MP}_{<\kappa-\text { dir. cl. }}\left(H_{\kappa^{+}}\right), \mathrm{MP}_{\mathrm{Col}(\omega)}\left(H_{\omega} \cup\{\omega\}\right)$ (note that the parameters allowed in the latter principle are definable, hence not necessary) and $\mathrm{MP}_{\mathrm{Col}(\omega)}\left(H_{\omega^{+}}\right)$ were analyzed in [Lei04] in terms of consistency strength. I shall quote some of the results obtained there, when they fit in.

It should be pointed out that I allow for $\kappa$ to be equal to $\omega$, in which case the restriction to $<\kappa$-closed or $<\kappa$-directed-closed forcings becomes vacuous, and so, the principle $\mathrm{MP}_{<\omega \text {-closed }}\left(H_{\omega} \cup\{\omega\}\right)$ is precisely the principle MP from [Ham03b]. Note that in case $\kappa=\omega_{1}$, the classes of $<\kappa$-closed and $<\kappa$-directedclosed forcings coincide.

For the rest of this section, I shall focus on the lightface principles $\mathrm{MP}_{<\dot{\kappa} \text {-closed }}\left(H_{\dot{\kappa}} \cup\{\dot{\kappa}\}\right), \mathrm{MP}_{<\dot{\kappa}-\text { dir. cl. }}\left(H_{\dot{\kappa}} \cup\{\dot{\kappa}\}\right)$ and $\mathrm{MP}_{\mathrm{Col}(\dot{\kappa})}\left(H_{\dot{\kappa}} \cup\{\dot{\kappa}\}\right)$.

Let's begin by analyzing the relationships between the principles introduced, in terms of implications. The following folkloristic facts will be crucial.

Lemma 2.2 Let $\kappa$ be a regular cardinal and $\lambda>\kappa$ a cardinal with $\lambda=\lambda^{<\kappa}$. Then there is a dense subset $\Delta$ of $\operatorname{Col}(\kappa, \lambda)$ such that if $\mathbb{P}$ is a separative $<\kappa$ closed partial order with $\overline{\overline{\mathbb{P}}}=\lambda$ and $\mathbb{1} \Vdash_{\mathbb{P}}(\overline{\bar{\lambda}}=\kappa)$, then there is a dense subset $D$ of $\mathbb{P}$ with $\operatorname{Col}(\kappa, \lambda) \uparrow \Delta \cong \mathbb{P}\lceil D$, i.e., $\operatorname{Col}(\kappa, \lambda)$ and $\mathbb{P}$ are forcing-equivalent.

Proof. This is an adaptation of the proof of the special case where $\kappa=\omega$ from [Jec03]. Let $\Delta=\{p \in \operatorname{Col}(\kappa, \lambda) \mid \exists \alpha<\lambda \quad \operatorname{dom}(p)=\alpha+1\}$, and let $\dot{f}$ be a $\mathbb{P}$-name such that $\mathbb{1} \Vdash_{\mathbb{P}} \dot{f}$ is a surjection from $\kappa$ onto $\Gamma$, where $\Gamma$ is the canonical name for the generic filter. By recursion on the length of $p \in{ }^{<\kappa} \lambda$, one can now define the desired isomorphism $\pi$, along with a sequence $\left\langle W_{p} \mid p \in{ }^{<\kappa} \lambda\right\rangle$, so that

- $\pi(p)$ is defined if $|p|$ is a successor ordinal,

- $W_{p}$ is a set of cardinality $\lambda$ s.t.

- $W_{p}$ is a maximal antichain in the restriction of $\mathbb{P}$ to the set of conditions which are lower bounds for $\{\pi(p\lceil(\gamma+1))|\gamma+1 \leq| p \mid\}$,

- every condition in $W_{p}$ decides the value of $\dot{f}(\check{\delta})$, where $\delta=|p|$,

- $W_{p}=\left\{\pi\left(p^{\curlyvee}\langle\alpha\rangle\right) \mid \alpha<\lambda\right\}$.

The construction is straightforward. It follows that $W_{\alpha}=\bigcup_{|p|=\alpha} W_{p}$ is a maximal antichain in $\mathbb{P}$.

It is clear that $\pi$ is an isomorphism between $\operatorname{Col}(\kappa, \lambda) \uparrow \Delta$ and its range, so it suffices to show that the latter is dense in $\mathbb{P}$. So let $q \in \mathbb{P}$ be given. Let $q^{\prime} \leq q$ and $\alpha<\kappa$ be such that $q^{\prime} \Vdash_{\mathbb{P}} \dot{f}(\check{\alpha})=\check{q}$ - note that $q \Vdash_{\mathbb{P}} \check{q} \in \Gamma$, so $\alpha$ and $q^{\prime}$ exist. Now since $W_{\alpha}$ is a maximal antichain in $\mathbb{P}$, there is some element of it which is compatible with $q^{\prime}$. Let this element be $\pi(p)$ (so $\left.|p|=\alpha+1\right)$. Since $\pi(p)$ is in $W_{\alpha}$, it decides the value of $\dot{f}(\check{\alpha})$, and since it is compatible with $q^{\prime}$, it decides it the same way that $q^{\prime}$ decided it, so $\pi(p) \Vdash_{\mathbb{P}} \dot{f}(\check{\alpha})=\check{q}$. In particular, $\pi(p) \Vdash \check{q} \in \Gamma$, and so, by separativity of $\mathbb{P}, \pi(p) \leq q$. 
Corollary 2.3 Let $\mathbb{P}$ be a $<\kappa$-closed notion of forcing, where $\kappa$ is regular. Then if $\lambda \geq \overline{\overline{\mathbb{P}}}$ and $\lambda^{<\kappa}=\lambda$,

$$
(\mathbb{P} \times \operatorname{Col}(\kappa, \lambda)) \uparrow D \cong \operatorname{Col}(\kappa, \lambda) \uparrow \Delta,
$$

for some dense set $D$ and the dense set $\Delta$ from Lemma 2.2.

So using terminology from [Lei04], $\operatorname{Col}(\kappa)$ absorbs the class of all $<\kappa$-closed forcings.

Corollary 2.4 Let $\kappa<\lambda$ be cardinals such that for all $\mu<\lambda, \mu^{<\kappa}<\lambda$. Let $\mathbb{P}$ be $a<\kappa$-closed notion of forcing with $\overline{\overline{\mathbb{P}}}<\lambda$. Then $\mathbb{P} \times \operatorname{Col}(\kappa,<\lambda)$ is forcing equivalent to $\operatorname{Col}(\kappa,<\lambda)$.

Proof. Let $\overline{\overline{\mathbb{P}}}=\mu<\lambda$. Then $\mu^{\prime}:=\mu^{<\kappa}<\lambda$, and $\mu^{\prime<\kappa}=\mu^{\prime}$. So writing $\cong_{f}$ for forcing equivalence, $\operatorname{Col}\left(\kappa,<\mu^{\prime}+1\right) \cong_{f} \operatorname{Col}\left(\kappa, \mu^{\prime}\right) \cong \mathbb{P} \times \operatorname{Col}\left(\kappa,<\mu^{\prime}+1\right)$. So

$$
\begin{aligned}
\mathbb{P} \times \operatorname{Col}(\kappa,<\lambda) & \cong_{f} \quad \mathbb{P} \times \operatorname{Col}\left(\kappa,<\mu^{\prime}+1\right) \times \operatorname{Col}\left(\kappa,\left(\mu^{\prime}+1, \lambda\right)\right) \\
& \cong_{f} \operatorname{Col}\left(\kappa,<\mu^{\prime}+1\right) \times \operatorname{Col}\left(\kappa,\left(\mu^{\prime}+1, \lambda\right)\right) \\
& \cong_{f} \quad \operatorname{Col}(\kappa,<\lambda) .
\end{aligned}
$$

Here, I wrote $\operatorname{Col}(\kappa,(\alpha, \beta))$ for the notion of forcing which collapses all ordinals in the interval $(\alpha, \beta)$ to $\kappa$.

I shall frequently make use of the previous lemma and its corollaries, without referring to them explicitly.

\section{Lemma 2.5}

$$
\begin{aligned}
& \mathrm{ZFC}+\mathrm{MP}_{\mathrm{Col}(\dot{\kappa})}\left(H_{\dot{\kappa}} \cup\{\dot{\kappa}\}\right) \\
& \vdash \quad \mathrm{ZFC}+\mathrm{MP}_{<\dot{\kappa}-\text { dir. cl. }}\left(H_{\dot{\kappa}} \cup\{\dot{\kappa}\}\right) \\
& \vdash \quad \mathrm{ZFC}+\mathrm{MP}_{<\dot{\kappa}-\text { closed }}\left(H_{\dot{\kappa}} \cup\{\dot{\kappa}\}\right) .^{2}
\end{aligned}
$$

Proof. It would be possible to use [Lei04, Cor. 2.7] here as a black-box, since $\mathrm{Col}(\kappa)$ absorbs all $<\kappa$-directed-closed forcings, and since the class of all $<\kappa$ directed-closed forcings absorbs that of all $<\kappa$-closed forcings (the latter is true since all forcings in $\operatorname{Col}(\kappa)$ are $<\kappa$-directed-closed). Here is the argument:

Let's work in a universe where $\dot{\kappa}$ is interpreted as $\kappa$, to eliminate the dot. In order to show that $\mathrm{MP}_{<\kappa-\text { dir. cl. }}\left(H_{\kappa} \cup\{\kappa\}\right) \Longrightarrow \mathrm{MP}_{<\kappa-\text { closed }}\left(H_{\kappa} \cup\{\kappa\}\right)$, it clearly suffices to show that if a statement $\varphi$, possibly involving the parameter

${ }^{2}$ In favor of readability, I shall in the future just write

$$
\mathrm{MP}_{\mathrm{Col}(\dot{\kappa})}\left(H_{\dot{\kappa}} \cup\{\dot{\kappa}\}\right) \Longrightarrow \mathrm{MP}_{<\dot{\kappa}-\text { dir. cl. }}\left(H_{\dot{\kappa}} \cup\{\dot{\kappa}\}\right) \Longrightarrow \mathrm{MP}_{<\dot{\kappa}-\text { closed }}\left(H_{\dot{\kappa}} \cup\{\dot{\kappa}\}\right)
$$

in order to express the above. 
$\kappa$ (and the proof goes thru even if $\varphi$ contains other parameters), is $<\kappa$-closedforceably necessary, then it is $<\kappa$-directed-closed-forceably necessary as well. This can be seen as follows: Let $\mathbb{P}$ be a $<\kappa$-closed notion of forcing making $\varphi<\kappa$-closed-necessary. Then $\mathbb{P}$ forces that it is $<\kappa$-closed-necessary that $\varphi$ is $<\kappa$-closed-necessary. Let $\delta=\overline{\overline{\mathbb{P}}}$ and let $\mathbb{Q}=\operatorname{Col}(\kappa, \theta)$, where $\theta \geq \delta$ and

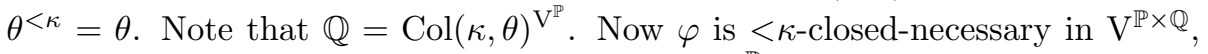
since this is a $<\kappa$-closed forcing extension of $\mathrm{V}^{\mathbb{P}}$. But $\mathbb{P} \times \mathbb{Q}$ is a $<\kappa$-closed forcing of size $\theta$ which collapses $\theta$ to $\kappa$. So a dense subset of it is isomorphic to $\operatorname{Col}(\kappa, \theta)=\mathbb{Q}$. But the latter forcing is $<\kappa$-directed-closed. So it is $<\kappa$ directed-closed-forceable that $\varphi$ is $<\kappa$-closed-necessary, and hence it is also $<\kappa$-directed-closed-forceable that $\varphi$ is $<\kappa$-directed-closed-necessary. In short, $\varphi$ is $<\kappa$-directed-closed-forceably necessary, as was to be shown.

The proof that $\mathrm{MP}_{\mathrm{Col}(\dot{\kappa})}\left(H_{\dot{\kappa}} \cup\{\dot{\kappa}\}\right) \Longrightarrow \mathrm{MP}_{<\kappa-\text { dir. cl. }}\left(H_{\kappa} \cup\{\kappa\}\right)$ is analogous: One shows that if $\varphi$ is $<\kappa$-directed-closed-forceably necessary, then it is $\operatorname{Col}(\kappa)$-forceably necessary. The point is that every $\operatorname{Col}(\kappa,<\delta)$ is $<\kappa$-directedclosed.

Part of the special case of the previous lemma, where $\kappa=\omega$, is also shown in [Lei04], namely that $\mathrm{MP}_{\mathrm{Col}(\omega)}\left(H_{\omega} \cup\{\omega\}\right)$ implies MP. It was pointed out by the referee that the following theorem holds, by methods of [Lei04], specifically Lemma 1.10, Theorem 1.11 and Corollary 1.12. I include a proof sketch for the reader's convenience.

Theorem 2.6 Let $\Gamma$ be the class of $<\kappa$-closed forcings, the class of $<\kappa$-directedclosed forcings or the class $\operatorname{Col}(\kappa)$. Then

$$
\operatorname{MP}_{\Gamma}(\{\kappa\}) \Longleftrightarrow \operatorname{MP}_{\Gamma}\left(H_{\kappa} \cup\{\kappa\}\right)
$$

Proof. Let's fix $\Gamma$, let's assume $\operatorname{MP}_{\Gamma}(\{\kappa\})$, and let's fix a formula $\varphi(x)$. The point is that the statement $\psi(\kappa)$ expressing "for any $a \in H_{\kappa} \cup\{\kappa\}$, if $\varphi(a)$ is $\Gamma$-forceably necessary, then $\varphi(a)$ holds" is $\Gamma$-forceably necessary. Note that once this is shown, the proof is complete, since $\operatorname{MP}_{\Gamma}(\{\kappa\})$ then implies that $\psi(\kappa)$ is true.

To see this, let $a \in H_{\kappa} \cup\{\kappa\}$. Let $\mathbb{P}_{a} \in \Gamma$ force $\varphi(a)$ to be $\Gamma$-necessary, if possible, and let $\mathbb{P}_{a}$ be trivial forcing if there is no such forcing. Now let $\mathbb{P}$ be the product of all $\mathbb{P}_{a}, a \in H_{\kappa} \cup\{\kappa\}$, with $<\kappa$-support. Then $\mathbb{P} \in \Gamma$, and the claim is that if $G$ is $\mathbb{P}$-generic, then $\psi(\kappa)$ holds in $\mathrm{V}[G]$. The point here is that $H_{\kappa}^{\mathrm{V}[G]}=H_{\kappa}^{\mathrm{V}}$. So if $a \in H_{\kappa} \cup\{\kappa\}^{\mathrm{V}[G]}$ and $\varphi(a)$ is $\Gamma^{\mathrm{V}[G]}$-forceably necessary over $\mathrm{V}[G]$ (by a forcing $\dot{\mathbb{Q}}^{G}$ ), then $\varphi(a)$ is also $\Gamma$-forceably necessary over $\mathrm{V}$ (by the forcing $\mathbb{P} * \dot{\mathbb{Q}})$. So $\mathbb{P}_{a}$ forces $\varphi(a)$ to be necessary. So it is necessary in $\mathrm{V}\left[G_{a}\right]$, where $G_{a}$ is the projection of $G$ onto its $a^{\text {th }}$ coordinate. So since $\mathrm{V}[G]$ is a forcing extension of $\mathrm{V}\left[G_{a}\right]$ by a forcing in $\Gamma^{\mathrm{V}\left[G_{a}\right]}, \varphi(a)$ is true in $\mathrm{V}[G]$. The same argument actually shows that $\psi(\kappa)$ stays true in every further forcing extension of $\mathrm{V}[G]$ by a forcing in $\Gamma^{\mathrm{V}[G]}$. 
So in case $\kappa$ is definable in a way that's absolute to generic extensions by forcings in $\Gamma$, then it even follows that $\operatorname{MP}_{\Gamma}(\emptyset)$ is equivalent to $\operatorname{MP}_{\Gamma}\left(H_{\kappa} \cup\{\kappa\}\right)$, where $\Gamma$ is any of the above classes of closed forcings.

Definition 2.7 Given two theories $T_{0}$ and $T_{1}$, both in the same language $\mathcal{L}^{*}$, which is the language of set theory, augmented by some additional constant symbols $\vec{c}$ and $\vec{d}$, I shall say that $T_{0}$ and $T_{1}$ are transitive model equiconsistent, locally in $\vec{c}$ if whenever $\langle M, \vec{a}, \vec{b}\rangle \models T_{0}$, with the constants $\vec{c}, \vec{d}$ interpreted as $\vec{a}, \vec{b}$, respectively and $M$ is countable and transitive, then there is a countable transitive model $N$ and a tuple $\vec{b}^{\prime}$ of elements of $N$ such that $M$ and $N$ have the same ordinals, and such that $\left\langle N, \vec{a}, \vec{b}^{\prime}\right\rangle \models T_{1}$, and vice versa.

I am going to show that the theory ZFC $+\mathrm{MP}_{<\dot{\kappa}-\text { closed }}\left(H_{\dot{\kappa}} \cup\{\dot{\kappa}\}\right)$ is locally in $\dot{\kappa}$ transitive model equiconsistent to the following theory in $\mathcal{L}_{\dot{\kappa}, \dot{\delta}}$ :

$$
\mathrm{ZFC}+\mathrm{V}_{\dot{\delta}} \prec \mathrm{V}+\dot{\kappa}<\dot{\delta}+\dot{\kappa} \text { is a regular cardinal . }
$$

The notation " $\mathrm{V}_{\dot{\delta}} \prec \mathrm{V}$ " stands for the scheme of sentences expressing that $\mathrm{V}_{\delta}$ is an elementary substructure of $\mathrm{V}$. So it consists of the sentences of the form $\forall \vec{x} \in \mathrm{V}_{\dot{\delta}} \quad\left(\varphi(\vec{x}) \Longleftrightarrow \varphi_{\mathrm{V}_{\dot{\delta}}}(\vec{x})\right)$, for every $\mathcal{L}$-formula $\varphi$ with free variables $\vec{x}$. I shall refer to an ordinal $\delta$ such that $\mathrm{V}_{\delta} \prec \mathrm{V}$ as an elementary rank. Let me point out that this scheme can be added to ZFC without increasing the strength of the theory, which follows by a straightforward application of Levy's Reflection scheme, together with the compactness theorem. This has already been pointed out in [Ham03b]. The scheme was merely added in order to arrive at transitive model equiconsistencies.

The following theorem is one direction of the equiconsistency I am aiming at. The converse is to come in the next section.

Theorem 2.8 Assume ZFC $+\mathrm{V}_{\dot{\delta}} \prec \mathrm{V}+\dot{\kappa}<\dot{\delta}+\dot{\kappa}$ is a regular cardinal . Then $\mathbb{1 1} \Vdash_{\mathrm{Col}(\kappa,<\delta)} \mathrm{MP}_{\mathrm{Col}(\kappa)}\left(H_{\kappa} \cup\{\kappa\}\right)$.

Proof. I omit the proof, as it is essentially contained in that of Theorem 2.10.

\subsection{Possible strengthenings: Boldface versions}

In this section, I shall examine the versions of the maximality principles at $\kappa$, with parameters from $H_{\kappa^{+}}$, i.e., $\mathrm{MP}_{<\kappa-\text { closed }}\left(H_{\kappa^{+}}\right), \mathrm{MP}_{<\kappa-\text { dir. cl. }}\left(H_{\kappa^{+}}\right)$and $\mathrm{MP}_{\mathrm{Col}(\kappa)}\left(H_{\kappa^{+}}\right)$. As before, I would like to point out that the case $\kappa=\omega$ is not excluded, and again, $\mathrm{MP}_{<\omega-\text { closed }}\left(H_{\omega_{1}}\right)$ is precisely the principle $\mathrm{MP}(\mathbb{R})$ from [Ham03b]. The dependencies between these principles reflect those between their lightface variants precisely:

Lemma 2.9 $\mathrm{MP}_{\mathrm{Col}(\dot{\kappa})}\left(H_{\dot{\kappa}^{+}}\right) \Longrightarrow \mathrm{MP}_{<\dot{\kappa}-\operatorname{dir} . \text { cl. }}\left(H_{\dot{\kappa}^{+}}\right) \Longrightarrow \mathrm{MP}_{<\dot{\kappa}-\operatorname{closed}}\left(H_{\dot{\kappa}^{+}}\right)$. 


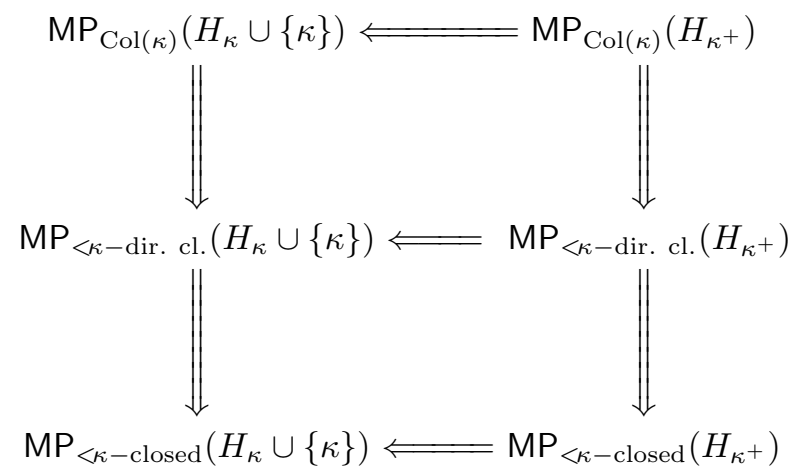

Figure 1: Implications between the maximality principles.

Proof. The argument establishing the lightface version works here as well.

So the diagram in Figure 1 shows the implications between the maximality principles introduced. It will turn out that none of these implications are reversible, in general, see Lemmas 4.3 and 4.4.

Consider the following theory in the language of set theory with additional constant symbols $\dot{\kappa}$ and $\dot{\delta}$ :

$$
\mathrm{ZFC}+\dot{\kappa}<\dot{\delta}+\dot{\kappa} \text { and } \dot{\delta} \text { are regular }+\mathrm{V}_{\dot{\delta}} \prec \mathrm{V} \text {. }
$$

I shall show that this theory is transitive model equiconsistent to each of the theories $\mathrm{MP}_{<\dot{\kappa} \text {-closed }}\left(H_{\dot{\kappa}^{+}}\right)+\dot{\delta}=\dot{\kappa}^{+}, \mathrm{MP}_{<\dot{\kappa}-\text { dir. cl. }}\left(H_{\dot{\kappa}^{+}}\right)+\dot{\delta}=\dot{\kappa}^{+}, \mathrm{MP}_{\mathrm{Col}(\dot{\kappa})}\left(H_{\dot{\kappa}^{+}}\right)$ $+\dot{\delta}=\dot{\kappa}^{+}$, locally in both constants $\dot{\kappa}$ and $\dot{\delta}$. The following is one direction of this equiconsistency. The converse will be proven at the end of the next section, see Corollary 3.10.

This can be viewed as a generalization of the special case for $\kappa=\omega$, which was proved by Hamkins in [Ham03b].

One may prove the following theorem by adapting arguments from [Ham03b], as in [Lei04, Thm. 6.10], the proof of which contains an argument establishing the version of the following theorem for $\mathrm{MP}_{<\kappa-\text { dir. cl. }}\left(H_{\kappa^{+}}\right)$. Here, I give a slightly shorter argument establishing a slightly stronger result.

Theorem 2.10 Assume that $\kappa<\delta, \kappa$ and $\delta$ are regular, and $\mathrm{V}_{\dot{\delta}} \prec \mathrm{V}$. Then $\mathrm{MP}_{\mathrm{Col}(\kappa)}\left(H_{\kappa^{+}}\right)$holds in $\mathrm{V}[G]$, where $G$ is $\mathrm{V}$-generic for $\mathbb{P}=\operatorname{Col}(\kappa,<\delta)$.

Proof. Assume that in $\mathrm{V}[G], \varphi(a)$ is $\operatorname{Col}(\kappa)$-forceably necessary, where $a \in$ $H_{\kappa^{+}}^{\mathrm{V}[G]}=\mathrm{V}_{\delta}[G]$. Let $\operatorname{Col}(\kappa,<\zeta)$ force $\varphi(a)$ to be $\operatorname{Col}(\kappa)$-necessary over $\mathrm{V}[G]$, where $\zeta$ may be picked as large as wished. Let $H$ be $\operatorname{Col}(\kappa,<\zeta)$-generic over $\mathrm{V}[G]$, so that $\varphi(a)$ is $\operatorname{Col}(\kappa)$-necessary in $\mathrm{V}[G][H]$. Let $\dot{a} \in \mathrm{V}_{\delta}$ be a $\mathbb{P}$-name s.t. $a=\dot{a}^{G}$. Pick $\theta<\delta$ large enough so that, setting $\overline{\mathbb{P}}=\operatorname{Col}(\kappa,<\theta)$ and $\bar{G}=G \cap \overline{\mathbb{P}}$, $a=\dot{a}^{\bar{G}}$. 
In $\mathrm{V}[\bar{G}]$, it is still the case that $\varphi(a)$ is forced to be $\operatorname{Col}(\kappa)$-necessary by $\operatorname{Col}(\kappa,<\zeta)$, because there is a $\operatorname{Col}(\kappa,<\zeta)$-generic filter over $\mathrm{V}[\bar{G}]$, so that $\mathrm{V}[G][H]=\mathrm{V}[\bar{G}]\left[H^{\prime}\right]$. So by homogeneity of $\operatorname{Col}(\kappa,<\zeta)$, 1 forces via $\operatorname{Col}(\kappa,<\zeta)$ over $\mathrm{V}[\bar{G}]$ that $\varphi(a)$ is $\operatorname{Col}(\kappa)$-necessary.

So since $\mathrm{V}_{\delta}[\bar{G}] \prec \mathrm{V}[\bar{G}]$, it is true in $\mathrm{V}_{\delta}[\bar{G}]$ that $\varphi(a)$ is $\operatorname{Col}(\kappa)$-forceably necessary. Let $\bar{\zeta}<\delta$ be such that $\operatorname{Col}(\kappa,<\bar{\zeta})$ forces $\varphi(a)$ to be $\operatorname{Col}(\kappa)$-necessary over $\mathrm{V}_{\delta}[\bar{G}]$. Again, $\bar{\zeta}$ may be picked as large as wished (below $\delta$ ). As before, it follows, setting $\tilde{\mathbb{P}}=\operatorname{Col}(\kappa,<\bar{\zeta})$ that $\mathrm{V}[G]=\mathrm{V}[\bar{G}][\tilde{G}]\left[G^{\prime}\right]$, where $\tilde{G}$ is $\tilde{\mathbb{P}}$-generic over $\mathrm{V}[\bar{G}]$ and $G^{\prime}$ is $\operatorname{Col}(\kappa,<\delta)$-generic over $\mathrm{V}[\bar{G}][\tilde{G}]$. And $\varphi(a)$ is $\operatorname{Col}(\kappa)$ necessary in $\mathrm{V}_{\delta}[\bar{G}][\tilde{G}]$. Since $\mathrm{V}_{\delta}[\bar{G}][\tilde{G}] \prec \mathrm{V}[\bar{G}][\tilde{G}]$, the same is true in $\mathrm{V}[\bar{G}][\tilde{G}]$.

But $\mathrm{V}[G]=\mathrm{V}[\bar{G}][\tilde{G}]\left[G^{\prime}\right]$ is a $\operatorname{Col}(\kappa,<\delta)$-generic extension of $\mathrm{V}[\bar{G}][\tilde{G}]$. Since $\varphi(a)$ is $\operatorname{Col}(\kappa)$-necessary in $\mathrm{V}[\bar{G}][\tilde{G}]$, it follows that $\varphi(a)$ is true in $\mathrm{V}[G]$, as claimed.

Let $\operatorname{Col}(\kappa,<$ inaccessible $)$ denote the class of forcings of the form $\operatorname{Col}(\kappa,<\theta)$, where $\theta>\kappa$ is inaccessible, and consider the corresponding maximality principle, together with the assertion that there are unboundedly many inaccessible cardinals. It is interesting to note that the proof of the previous theorem actually establishes that $\operatorname{Col}(\kappa,<\delta)$-generic extensions of $\mathrm{V}$ are models of that principle.

It is also clear that this principle implies $\operatorname{MP}_{\operatorname{Col}(\kappa)}\left(H_{\kappa} \cup\{\kappa\}\right)$. So one can ask the corresponding questions about the reversibility of these implications, both in the boldface and in the lightface context. Note that it also implies that $2^{\kappa}=\kappa^{+}$(since this is forceably necessary with respect to forcings in $\operatorname{Col}(\kappa,<$ inaccessible)). Contrasting this, I show in 4.11 that the value of $2^{\kappa}$ is not determined by $\mathrm{MP}_{<\kappa-\text { closed }}\left(H_{\kappa^{+}}\right)$.

\section{Consequences}

Before proving the converses to Theorems 2.8 and 2.10, let's draw some consequences of the maximality principles for $<\kappa$-closed forcings that I introduced. The reader should keep in mind that these principles follow from the corresponding principles for forcings in $\operatorname{Col}(\kappa)$ or for the class of $<\kappa$-directed-closed forcings.

Theorem 3.1 Assume $\mathrm{MP}_{<\kappa-\operatorname{closed}}\left(H_{\kappa} \cup\{\kappa\}\right)$, where $\kappa$ is an uncountable, regular cardinal. Then $\vee_{\kappa}$ holds.

Proof. It is well-known that by adding a subset of $\kappa$ with $<\kappa$-closed forcing, $\nabla_{\kappa}$ comes true. But once true, it cannot be destroyed again by $<\kappa$-closed forcing. This was observed by Jensen, see [DJ74]; it is even the case that any particular $\nabla_{\kappa}$-sequence will continue to be a $\nabla_{\kappa}$-sequence in any further forcing extension obtained by $<\kappa$-closed forcing. So $\nabla_{\kappa}$ is $<\kappa$-closed-forceably necessary, and hence true, by the maximality principle. 
Lemma 3.2 (Silver for $\kappa=\omega_{1}$ ) Let $\kappa$ be a regular uncountable cardinal, and let $T$ be a $\kappa$-tree. In case $\kappa$ is a limit cardinal, assume in addition that $T$ is slim. ${ }^{3}$ If $\mathbb{P}$ is a $<\kappa$-closed notion of forcing and $G$ is generic for $\mathbb{P}$, then, denoting the set of branches of $T$ by $[T]$, it follows that

$$
[T]=[T]^{\mathrm{V}[G]} .
$$

Proof. Assume $\dot{b}$ was a name for a new branch through $T$. Let $p$ force this. Then it is possible to construct sequences $\left\langle p_{t} \mid t \in{ }^{<\kappa} 2\right\rangle,\left\langle\gamma_{\alpha} \mid \alpha<\kappa\right\rangle$ and $\left\langle b_{t}\right|$ $\left.t \in{ }^{<\kappa} 2\right\rangle$ such that $s \subseteq t \Longrightarrow p_{t} \leq p_{s} \leq p, b_{t}$ is a branch of order-type $\gamma_{|t|}$, $p_{t} \Vdash \dot{b}\left\lceil\gamma_{|t|}=b_{t}, b_{t<\langle 0\rangle} \neq b_{t-\langle 1\rangle}\right.$, and $\left\langle\gamma_{\alpha} \mid \alpha<\kappa\right\rangle$ is a normal function. So for limit $\alpha$, the branches $\left\{b_{t}|| t \mid=\alpha\right\}$ give rise to $2^{\alpha}$ many nodes at level $\gamma_{\alpha}$ of $T$. Since $T$ is a $\kappa$-tree, this set has size less than $\kappa$, which keeps the construction going. But by the normality of the sequence $\vec{\gamma}$, one can now take a fixed-point $\alpha=\gamma_{\alpha}$ which is a limit. If $\kappa=\bar{\kappa}^{+}$is a successor cardinal, then such an $\alpha$ of cardinality $\bar{\kappa}$ can be chosen. In any case then, $T$ has $2^{\alpha}$ nodes at level $\alpha=\gamma_{\alpha}$, so that $T$ is not slim. But $\kappa$ can't be a successor either, because then by the choice of $\alpha, T$ would have $2^{\alpha} \geq \kappa$ many nodes at level $\alpha$, contradicting the assumption that $T$ is a $\kappa$-tree.

Theorem 3.3 Assume $\mathrm{MP}_{<\kappa-\text { closed }}(S)$, where $\kappa$ is a regular uncountable cardinal. Then there is no slim $\kappa$-Kurepa tree in $S$. If $\kappa$ is a successor, then there is no $\kappa$-Kurepa tree in $S$. So if $S=H_{\kappa^{+}}$, then there are no such trees at all.

Proof. Assume that $T \in S$ were a $\kappa$-Kurepa tree which is slim in case $\kappa$ is a limit cardinal. Let $\lambda=\overline{\overline{[T]}}$. Then forcing with $\operatorname{Col}(\kappa, \lambda)$ doesn't add branches to $T$, by Lemma 3.2 and collapses the cardinality of the set of branches through $T$ to $\kappa$. So $T$ is not a Kurepa-tree in the extension. And $T$ can never become a Kurepa-tree in any further forcing extension by $<\kappa$-closed forcing, by the same lemma. So $T$ is forceably necessarily not a Kurepa-tree. Since $T$ is allowed as a parameter in $\mathrm{MP}_{<\kappa-\text { closed }}(S)$, it follows that $T$ is not a Kurepa tree, a contradiction.

Definition 3.4 Let $\kappa$ be a regular cardinal, $n$ a natural number and $M$ a transitive set (usually either $\kappa$ or $H_{\kappa}$ ). Then $<\kappa$-closed-generic $\Sigma_{n}^{1}(M)$-absoluteness with parameters in $S$ is the statement that for any $\Sigma_{n}^{1}$-sentence $\varphi$ with predicate symbols $\vec{a}$, the following holds: Whenever $\vec{a} \in S \cap \mathcal{P}(M), \mathbb{P}$ is a $<\kappa$-closed notion of forcing and $G$ is $\mathbb{P}$-generic over $\mathrm{V}$, then

$$
(\langle M, \in, \vec{a}\rangle \models \varphi)^{\mathrm{V}} \Longleftrightarrow(\langle M, \in, \vec{a}\rangle \models \varphi)^{\mathrm{V}[G]},
$$

where it is understood that $\overrightarrow{\dot{a}}$ is interpreted in $M$ as $\vec{a}$. The case $S=\mathcal{P}(\kappa)$ is boldface $<\kappa$-closed-generic $\Sigma_{n}^{1}(M)$-absoluteness. If $\kappa=\omega$, then $<\kappa$-closed

\footnotetext{
${ }^{3}$ By a $\kappa$-tree, I mean a tree of height $\kappa$ all of whose levels have size less than $\kappa$, and by a slim $\kappa$-tree, I mean a $\kappa$ tree $T$ with the property that for any $\alpha<\kappa$, the $\alpha^{\text {th }}$ level of $T$ has size at most $\overline{\overline{\alpha+\omega}}$.
} 
generic $\Sigma_{n}^{1}(\omega)$-absoluteness with parameters in $S$ is referred to as generic $\Sigma_{n}^{1}$ absoluteness with parameters in $S$.

Lemma 3.5 (Silver) Let $\kappa$ be regular. Then boldface $<\kappa$-closed-generic $\Sigma_{1}^{1}(\kappa)$ absoluteness holds.

Proof. This is known, see [Kun80, p. 298, (I6)].

Theorem 3.6 Assume $\mathrm{MP}_{<\kappa-\text { closed }}(S)$, where $\kappa \in S$. Then $<\kappa$-closed-generic $\Sigma_{2}^{1}\left(H_{\kappa}\right)$-absoluteness with parameters in $S$ holds. So if $S=H_{\kappa^{+}}$, then boldface $<\kappa$-closed-generic $\Sigma_{2}^{1}\left(H_{\kappa}\right)$-absoluteness follows.

In case $\kappa=\omega$, i.e., if $\mathrm{MP}(S)$ holds, then $\Sigma_{3}^{1}$-absoluteness with parameters in $S$ follows, so that $\mathrm{MP}(\mathbb{R})$ implies boldface generic $\Sigma_{3}^{1}$-absoluteness.

Proof. First, observe that $H_{\kappa}$ has size $\kappa$. This is trivial if $\kappa=\omega$, and it follows from Theorem 3.1 otherwise. This means that the previous Lemma 3.5 can be improved to give boldface $<\kappa$-closed-generic $\Sigma_{1}^{1}\left(H_{\kappa}\right)$-absoluteness. In case $\kappa=\omega$, even generic boldface $\Sigma_{2}^{1}$-absoluteness holds (Shoenfield absoluteness).

In particular, this means that true $\Sigma_{2}^{1}\left(H_{\kappa}\right)$-statements $\left(\Sigma_{3}^{1}\right.$-statements in case $\kappa=\omega)$ with arbitrary parameters in $\mathcal{P}\left(H_{\kappa}\right)$ persist to $<\kappa$-closed-generic extensions. And this is not only true in $\mathrm{V}$, but also in any forcing extension of $\mathrm{V}$ obtained by $<\kappa$-closed forcing, since in any such extension, it will still be the case that $H_{\kappa}$ has size $\kappa$ - in fact, $H_{\kappa}$ doesn't change. So, in passing, this already shows one direction of the postulated absoluteness result.

Now let $G$ be generic over $\mathrm{V}$ for a $<\kappa$-closed forcing, let $\varphi$ be a $\Sigma_{2}^{1}$-formula (a $\Sigma_{3}^{1}$-formula in case $\kappa=\omega$ ), and let $\vec{a} \in S \cap \mathcal{P}\left(H_{\kappa}\right) \cap \mathrm{V}$. Let $\psi(\kappa, \vec{a})$ be the statement " $\left\langle H_{\kappa}, \in, \vec{a}\right\rangle \models \varphi$ ". Assume that $\psi(\kappa, \vec{a})$ holds in $\mathrm{V}[G]$. I have to show that it holds in $\mathrm{V}$ as well. But the initial observation shows that $\psi(\kappa, \vec{a})$ is true not only in $\mathrm{V}[G]$, but in any further forcing extension obtained by $<\kappa$-closed forcing. In other words, $\psi(\kappa, \vec{a})$ is $<\kappa$-closed-forceably necessary. Since the parameters used are in $S$, it follows by $\mathrm{MP}_{<\kappa-\text { closed }}(S)$ that $\psi(\kappa, \vec{a})$ is already true in $\mathrm{V}$.

Just to illustrate, let's note:

Theorem 3.7 If $\kappa=\bar{\kappa}^{+}$, where $2^{<\bar{\kappa}}=\bar{\kappa}$, and $\mathrm{MP}_{<\kappa-\text { closed }}\left(H_{\kappa} \cup\{\kappa\}\right)$ holds, then there is a $\kappa$-Souslin tree.

Proof. Under the hypothesis, a $\kappa$-Souslin tree can be added by a $<\kappa$-closed forcing - see [HT00]. In case $\kappa>\omega_{1}$, it is a variant of the Jech partial order to add an $\omega_{1}$-Souslin tree. One forces with $<\bar{\kappa}$-closed trees which will be segments of the generic Souslin tree. But once a Souslin tree is added, it is $<\kappa$-closednecessarily a Souslin tree, by Lemma 3.5 , since this is a $\Pi_{1}^{1}\left(H_{\kappa}\right)$-property of the tree.

An alternative way to see this is as follows: In the current situation, one can force $\diamond_{\kappa}\left(\mathrm{CF}_{\bar{\kappa}}\right)$ with $<\kappa$-closed forcing, and that principle is then necessary, and 
hence true. But under this assumption, together with $2^{<\bar{\kappa}}=\bar{\kappa}$, it is well-known that a $\kappa^{+}$-Souslin tree can be constructed - see [FH07].

The proof of the previous theorem suggests that the existence of certain highly rigid $\kappa$-Souslin trees might follow under the assumptions of that theorem. Indeed, Souslin trees that were generically added as described, exhibit rigidity degrees exceeding those introduced in [FH06], and some of them are $<\kappa$-closednecessary, once they are true. I shall give an example in Theorem 3.15.

Theorem 3.8 Assume $\mathrm{MP}_{<\kappa-\operatorname{closed}}\left(H_{\kappa^{+}}\right)$. Then $\kappa^{+}$is inaccessible in L, and $L_{\kappa^{+}} \prec L$.

Proof. It suffices to show that $L_{\kappa^{+}} \prec L$, since $\kappa^{+}$is clearly regular in $L$. It follows then that $\kappa^{+}$is inaccessible in $L$, for it then has to be a limit cardinal in $L$ : In $L$, there are arbitrarily large cardinals, so the same is true in $L_{\kappa^{+}}$.

The proof of that $L_{\kappa^{+}} \prec L$ parallels that of [Ham03b, Lemma 10.1]. Namely, one just verifies the Tarski-Vaught criterion:

Assume $\vec{a} \in L_{\kappa^{+}}$and $L \models \exists z \quad \varphi(z, \vec{a})$. Consider the statement "the least ordinal $\gamma$ such that there is a $b \in L_{\gamma}$ with $\varphi_{L}(b, \vec{a})$ has cardinality at most $\kappa$ ". The parameters in that statement come from $H_{\kappa^{+}}$, and it is $<\kappa$-closed forceably necessary. So it is true, which means that there is a witness $b$ for the existential statement in $L_{\kappa^{+}}$.

\subsection{Equiconsistencies}

Note that Theorem 3.8 gives the converse of Theorem 2.10. The converse of the theorem concerning the lightface principle is given by the following Lemma.

Lemma 3.9 Let $M$ be a set-sized transitive model of ZFC $+\mathrm{MP}_{<\kappa-\text { closed }}(\{\kappa\})$. Let $\delta$ be the supremum of the ordinals that are definable over $L^{M}$ in the parameter $\kappa$. Then $\delta<$ On $\cap M$. So $\left\langle L^{M}, \kappa, \delta\right\rangle$ is a model of the theory (with two additional constant symbols) expressing: " $\kappa$ is regular, $\kappa<\delta$ and $\mathrm{V}_{\delta} \prec \mathrm{V}$."

Proof. First, $\delta \leq\left(\kappa^{+}\right)^{M}$, and in particular, $\delta \in M$. This is because if $\gamma$ is definable over $L^{\bar{M}}$ from $\kappa$, then $\gamma<\left(\kappa^{+}\right)^{M}$. For let $\gamma$ be the unique $\xi$ such that $L^{M} \models \varphi[\xi, \kappa]$. Then the statement "the unique ordinal $\xi$ such that $\varphi[\xi, \kappa]$ holds has cardinality $\leq \kappa$ " is $<\kappa$-closed-forceably necessary over $M$, and hence true in $M$, by $\mathrm{MP}_{<\kappa-\operatorname{closed}}(\{\kappa\})$ in $M$.

The second point is that $L_{\delta} \prec L^{M}$. This is true in general, and doesn't use the maximality principle at all. It is shown by verifying the Tarski-Vaught criterion. So let $L^{M} \models \exists x \quad \varphi[x, \vec{a}]$, where $\vec{a} \in L_{\delta}$. Let $\vec{a} \in L_{\alpha}$ where $\alpha$ is definable from $\kappa$ over $M$. Then by replacement in $M$, there is a least $\beta \in M$ such that for all $\vec{a}^{\prime} \in L_{\alpha}$, if there is some $b \in L^{M}$ such that $L \models \varphi\left[b, \vec{a}^{\prime}\right]$, then there is some such $b \in L_{\beta}$. This $\beta$ is then definable over $M$ using $\kappa$ as a parameter I just gave a definition. So $\beta<\delta$, and hence there is some $b \in L_{\delta}$ such that 
$L^{M} \models \varphi[b, \vec{a}]$, as wished.

Summarizing, Theorems 2.8, 2.10, 3.8 and Lemma 3.9 together show:

Corollary 3.10 The following equiconsistencies hold:

1. The theory $\mathrm{ZFC}+\mathrm{MP}_{<\dot{\kappa} \text {-closed }}\left(H_{\dot{\kappa}} \cup\{\dot{\kappa}\}\right)$ is transitive model equiconsistent to

$$
\mathrm{ZFC}+\dot{\kappa} \text { is regular }+\dot{\kappa}<\dot{\delta}+\mathrm{V}_{\dot{\delta}} \prec \mathrm{V} \text {, }
$$

locally in $\dot{\kappa}^{4}$

2. The theory $\mathrm{ZFC}+\mathrm{MP}_{<\dot{\kappa} \text {-closed }}\left(H_{\dot{\kappa}^{+}}\right)+\dot{\delta}=\dot{\kappa}^{+}$is transitive model equiconsistent to the theory

$$
\text { ZFC }+\dot{\kappa} \text { is regular }+\dot{\kappa}<\dot{\delta}+\dot{\delta} \text { is regular }+\mathrm{V}_{\dot{\delta}} \prec \mathrm{V},
$$

locally in $\dot{\kappa}$ and $\dot{\delta}$.

\subsection{Closed maximality principles at large cardinals}

I close this section with some results concerning the compatibility of the closed maximality principles at $\kappa$ with large cardinal properties of $\kappa$. Let's start with the following lemma.

Lemma 3.11 Let $\varphi(\dot{\kappa})$ express one of the following statements in the language of set theory, augmented by the constant symbol $\dot{\kappa}: \dot{\kappa}$ is inaccessible, Mahlo, subtle, Woodin.

1. The theory

$$
\mathrm{ZFC}+\mathrm{MP}_{<\dot{\kappa}-\text { closed }}\left(H_{\dot{\kappa}} \cup\{\dot{\kappa}\}\right)+\varphi(\dot{\kappa})
$$

is transitive model equiconsistent to the theory expressing

$$
\mathrm{ZFC}+\text { " } \dot{\kappa} \text { is regular" }+\dot{\kappa}<\dot{\delta}+\mathrm{V}_{\dot{\delta}} \prec \mathrm{V}+\varphi(\dot{\kappa}),
$$

locally in $\dot{\kappa}$.

2. The theory

$$
\mathrm{ZFC}+\mathrm{MP}_{<\dot{\kappa}-\text { closed }}\left(H_{\dot{\kappa}^{+}}\right)+\dot{\delta}=\dot{\kappa}^{+}+\varphi(\dot{\kappa})
$$

is transitive model equiconsistent to the theory

$$
\text { ZFC }+\dot{\kappa}<\dot{\delta}+" \dot{\kappa} \text { and } \dot{\delta} \text { are regular" }+\mathrm{V}_{\dot{\delta}} \prec \mathrm{V}+\varphi(\dot{\kappa}),
$$

locally in $\dot{\kappa}$ and $\dot{\delta}$.

${ }^{4}$ It should be emphasized again that the consistency strength of these theories is no more than that of ZFC. 
Proof. I deal with part 2 first. Starting from a model of the second theory, where this theory is realized by $\kappa$ and $\delta$, forcing with $\operatorname{Col}(\kappa,<\delta)$ yields a model of $\mathrm{MP}_{<\kappa-\text { closed }}\left(H_{\kappa^{+}}\right)+\delta=\kappa^{+}$. But since this forcing is $<\kappa$-closed, $\varphi(\kappa)$ is also preserved: Inaccessibility is obviously preserved, and Mahloness as well as subtlety and Woodinness are $\Pi_{1}^{1}(\kappa)$ properties, and hence preserved, by Lemma 3.5 .

Vice versa, given a model $M$ in which $\mathrm{MP}_{<\kappa-\text { closed }}\left(H_{\kappa^{+}}\right)+\delta=\kappa^{+}+\varphi(\kappa)$ holds, where $\varphi(\kappa)$ expresses that $\kappa$ is Mahlo or subtle, then $L^{M}$ is a model of the second theory, since inaccessibility, Mahloness and subtlety pass down from $\mathrm{V}$ to $L$. If $\varphi(\kappa)$ expresses that $\kappa$ is Woodin, then set $N=(L[A])^{M}$, where $A$ is some subset of $\kappa$ which codes $\mathrm{V}_{\kappa}=H_{\kappa}$. Obviously, $N$ is a model of the second theory. The point here is that the proof of Theorem 3.8 goes through for $L[A]$ instead of $L$, since $L[A]$ has a definition which is absolute for the forcings in question, and $A$ is allowed as a parameter in the maximality principle.

Now let's turn to the lightface versions of this, i.e., to part 1. It is clear how to obtain a model of ZFC $+\operatorname{MP}_{\mathrm{Col}(\kappa)}\left(H_{\kappa} \cup\{\kappa\}\right)$ starting with a model of the second theory, as before by forcing with $\operatorname{Col}(\kappa,<\delta) ; \varphi(\kappa)$ is preserved in each case, by the reasons given. The other direction also works as before, except in case $\varphi(\kappa)$ expresses that $\kappa$ is Woodin. In that case, starting with a model $M$ of $\mathrm{MP}_{<\kappa \text {-closed }}\left(H_{\kappa} \cup\{\kappa\}\right)+\varphi(\kappa)$, the model $N$ to work with is $L^{M}\left(\mathrm{~V}_{\kappa}^{M}\right)[G]$, where $G$ is $\mathbb{P}:=\operatorname{Col}\left(\kappa, \mathrm{V}_{\kappa}\right)^{M}$-generic over $M$. Then $N$ is a model of ZFC, since the forcing added a well-ordering of $\mathrm{V}_{\kappa}$, and it has the desired properties. The details of this argument are as follows: Let $\delta$ be the supremum of the ordinals which are definable in the parameter $\kappa$ over $L\left(\mathrm{~V}_{\kappa}\right)^{M}$. The proof of Lemma 3.9 yields that $\delta \in M$ (the point here is that the canonical definition of $L\left(\mathrm{~V}_{\kappa}\right)^{M}$, using merely $\kappa$ as a parameter, is absolute to $<\kappa$-closed forcing extensions of $M)$, and that $L_{\delta}\left(\mathrm{V}_{\kappa}\right)^{M}=\mathrm{V}_{\delta}^{L\left(\mathrm{~V}_{\kappa}\right)^{M}} \prec L\left(\mathrm{~V}_{\kappa}\right)^{M}$. It follows that $\mathbb{P} \in L_{\delta}\left(\mathrm{V}_{\kappa}\right)$, since $\mathbb{P}$ is definable in $L\left(\mathrm{~V}_{\kappa}\right)^{M}$ in the parameter $\kappa$. So

$$
\mathrm{V}_{\delta}^{N}=L_{\delta}\left(\mathrm{V}_{\kappa}\right)[G] \prec L\left(\mathrm{~V}_{\kappa}\right)[G]=N .
$$

Since the statement " $\kappa$ is Woodin" is $\Pi_{1}^{1}\left(H_{\kappa}\right)$, it remains true in $M[G]$; note that the proof of this fact uses the axiom of choice, so that one couldn't directly argue that $\kappa$ is Woodin in $N$. But $\kappa$ is Woodin in $N$, since $N \subseteq M[G]$, $\mathrm{V}_{\kappa}^{N}=\mathrm{V}_{\kappa}^{M[G]}$ and $\kappa$ is Woodin in $M[G]$.

The following was observed by Leibman in [Lei04], with $\mathrm{MP}_{\mathrm{Col}(\kappa)}\left(H_{\kappa^{+}}\right)$replaced by $\mathrm{MP}_{<\kappa-\text { dir. cl. }}\left(H_{\kappa^{+}}\right)$. The same proof actually shows a slightly stronger result, and since it is very short, I shall give here.

Lemma 3.12 Suppose $\kappa$ is supercompact and $\kappa<\delta$, where $\delta$ is an inaccessible cardinal such that $\mathrm{V}_{\delta} \prec \mathrm{V}$. Then there is a forcing extension $\mathrm{V}[G]$ of $\mathrm{V}$ in which $\mathrm{MP}_{\mathrm{Col}(\kappa)}\left(H_{\kappa^{+}}\right)$holds and in which $\kappa$ is still supercompact.

Proof. This is achieved by a two-step extension. First, force with the Laver preparation forcing. This is a forcing of size $\kappa$ which is $\kappa$-c.c., and which results 
in an extension $\mathrm{V}[H]$ such that $\kappa$ is supercompact in any further extension of $\mathrm{V}[H]$ by a $<\kappa$-directed closed forcing. Since the Laver preparation is a small forcing, it follows that $\mathrm{V}_{\delta}^{\mathrm{V}[H]} \prec \mathrm{V}[H]$. So now it is possible to force over $\mathrm{V}[H]$ with $\operatorname{Col}(\kappa,<\delta)$, which yields an extension $\mathrm{V}[H][I]=\mathrm{V}[G]$, in which $\operatorname{MP}_{\operatorname{Col}(\kappa)}\left(H_{\kappa^{+}}\right)$ holds. But this collapse is $<\kappa$-directed-closed, so $\kappa$ is supercompact in $\mathrm{V}[G]$.

For the version of this lemma about the lightface principle, one can drop the requirement that $\delta$ be inaccessible.

Up to now, the only way I know how to arrive at a model in which $\kappa$ is weakly compact and a boldface closed maximality principle holds at $\kappa$ is the one described in the proof of Lemma 3.12, i.e., an upper bound for the consistency strength is $\kappa$ is supercompact $+\kappa<\delta$, where $\delta$ is inaccessible and $\mathrm{V}_{\delta} \prec \mathrm{V}$. So one arrives at the following questions:

\section{Question 3.13}

1. What is the consistency strength of $\mathrm{MP}_{<\kappa-\text { closed }}\left(H_{\kappa} \cup\{\kappa\}\right)$ holding at a weakly compact cardinal?

2. What is the strength of $\mathrm{MP}<\kappa-\operatorname{closed}\left(H_{\kappa^{+}}\right)$holding at a weakly compact $\kappa$ ?

Let's call a weakly compact cardinal $\kappa$ that remains weakly compact in any forcing extension by a $<\kappa$-closed partial order an indestructibly weakly compact cardinal. The following gives an answer to the first part of the question, if one accepts that large cardinal property of $\kappa$ as a measure of consistency strength.

Lemma 3.14 Let $\mathcal{L}_{\dot{\kappa}, \dot{\delta}}$ be the language of set theory with additional constant symbols $\dot{\kappa}$. Then the following theories are equiconsistent:

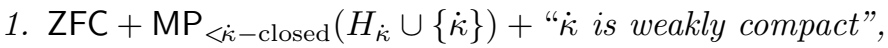

2. ZFC + " $\dot{\kappa}$ is indestructibly weakly compact".

Proof. To see that the consistency of the theory in 1 . implies that of the theory in 2., note that the statement that $\kappa$ is weakly compact is expressed by a $\Pi_{2}^{1}$-formula over $H_{\kappa}$. But by Theorem 3.6, MP $<\kappa$-closed $\left(H_{\kappa} \cup\{\kappa\}\right)$ implies $\Sigma_{2}^{1}\left(H_{\kappa}\right)$-absoluteness, and hence that $\kappa$ is indestructibly weakly compact.

For the converse, note that if the theory mentioned in 2. is consistent, then so is the theory formulated in the language with additional constant symbol $\dot{\delta}$ which consists of the theory in 2 , , plus " $\dot{\kappa}<\dot{\delta}+\mathrm{V}_{\dot{\delta}} \prec \mathrm{V}$ ". Forcing with $\operatorname{Col}(\kappa,<\delta)$ over a model of that theory produces a model of the first theory.

Of course, in terms of consistency strength, in the previous lemma, it doesn't matter whether the maximality principle considered in 1. refers to all forcings that are $<\kappa$-closed, or just to the $<\kappa$-directed-closed ones, or only to forcings in $\operatorname{Col}(\kappa)$.

There is more to be said on the consistency strength of an indestructibly weakly compact cardinal: By results from [AH01], if $\kappa$ is indestructibly weakly 
compact, and this indestructibility was achieved by forcing that has a closure point below $\kappa$ (see Definition 6.5), then $\kappa$ was supercompact in the ground model.

Also, by results of Schimmerling and Steel (see [SS99]), if $K$ exists and $\kappa$ is weakly compact, then $\kappa$ is weakly compact in $K$ and $\kappa^{+K}=\kappa^{+}$. So $K$ cannot exist if $\kappa$ is $<\kappa$-closed-indestructibly weakly compact, since $K$ would be absolute to forcing extensions, in particular to those in which $\kappa^{+}$is collapsed. Using this fact, methods of Woodin can be used to run a core model induction on this assumption.

Finally, the fact that after collapsing $\kappa^{+}$to $\kappa,\left(\kappa^{+}\right)^{\mathrm{HOD}}<\kappa^{+}$in the generic extension, shows that in that model, there is no extender model at all, which satisfies weak covering at $\kappa$. This is because any such model is contained in HOD. At the same time, $\kappa$ remains weakly compact in the generic extension, by assumption. So this is a model in which $\kappa$ is weakly compact, but there is no extender model at all which satisfies weak covering. This was observed by the author. Subsequently, Ralf Schindler noticed that this implies the existence of a non-domestic premouse. The latter observation uses recent core model theoretic methods, in particular the Jensen stack. It is proven in [JSSS07]. A non-domestic mouse is an iterable model of $\mathrm{ZFC}^{-}$with a cardinal $\kappa$ which is simultaneously a limit of Woodin cardinals, a limit of cardinals which are strong up to $\kappa$, and externally measurable. The existence of such a mouse is stronger than the $A D_{\mathbb{R}}$ hypothesis.

\subsection{An application to the automorphism tower problem}

The proof of Theorem 3.7 suggested that $\mathrm{MP}_{<\kappa-\text { closed }}\left(H_{\kappa} \cup\{\kappa\}\right)$ might have consequences concerning the existence of $\kappa$-trees with special automorphism properties. I will confirm this in the following, giving an example.

First, I would like to give some background on the automorphism tower: Given a centerless group $G$, its automorphism tower is obtained by iteratively computing its automorphism group, the automorphism group of that group, and so on transfinitely. Each group maps naturally into the next by sending a group element to the inner automorphism which conjugates by that element. At limits, one forms the direct limit of the system constructed so far.

$$
G \rightarrow \operatorname{Aut}(G) \rightarrow \operatorname{Aut}(\operatorname{Aut}(G)) \rightarrow \cdots \rightarrow G_{\alpha} \rightarrow G_{\alpha+1} \rightarrow \cdots
$$

The tower terminates when a fixed point is first reached, a group that is isomorphic to its automorphism group by the natural map, and this terminating ordinal is the height of the tower. For much more on the automorphism tower problem, the reader is referred to [Tho].

In [HT00], it was shown that it is consistent to have ZFC together with the following statement, for every cardinal $\lambda$ :

$(\star)_{\lambda}$ For any ordinal $\alpha<\lambda$, there is a centerless group $G$ the height of whose automorphism tower is $\alpha$, but given any nonzero $\beta<\lambda$, there is a notion 
of forcing $\mathbb{P}$, which preserves cofinalities and cardinalities, such that the height of the automorphism tower of the same group is $\beta$ in every generic extension by $\mathbb{P}$.

It was shown there that the previous statement follows from a combinatorial property which I am going to state presently, using terminology from [FH07]. Namely, say that a sequence $\left\langle T^{\alpha} \mid \alpha<\lambda\right\rangle$ of $\lambda$-Souslin trees is able to realize an equivalence relation $E$ on $\lambda$, if there is a notion of forcing $\mathbb{P}_{E}$ with the following properties:

1. $\mathbb{P}_{E}$ preserves cardinals and cofinalities, and has cardinality $\lambda$,

2. $\mathbb{P}_{E}$ doesn't add new sequences of elements of the ground model of length less than $\lambda$,

3. After forcing with $\mathbb{P}_{E}$ each of the trees $T^{\alpha}$, for $\alpha<\lambda$, is rigid,

4. In any extension $\mathrm{V}[G]$ obtained by forcing with $\mathbb{P}_{E}, E$ is realized, in the sense that $T^{\mu} \cong T^{\nu}$ iff $\mu E \nu$, for $\mu, \nu<\lambda$.

The statement implying $(\star)_{\lambda}$ (say, for $\lambda$ regular) is that there is a $\lambda$-sequence $\vec{T}$ of rigid, pairwise non-isomorphic $\lambda$-Souslin trees which is able to realize every equivalence relation on $\lambda$. Indeed, this is more than needed in order to derive $(\star)_{\lambda}$; see [FH07]. Namely, it suffices that $\vec{T}$ is able to realize all equivalence relations $E$ of the form $E=\{\langle\gamma, \delta\rangle \mid \gamma=\delta$ or $\{\gamma, \delta\}=\{0, \alpha\}\}$ or of the form $E=\{\langle\gamma, \delta\rangle \mid \gamma=\delta$ or $\gamma, \delta<\alpha\}$, for some $\alpha<\kappa^{+}$. So these equivalence relations just identify 0 and $\alpha$, or all the ordinals below $\alpha$. Let's call such equivalence relations simple. I shall prove:

Theorem 3.15 Assume that $\kappa$ is regular, $\mathrm{MP}_{<\kappa^{+}-\text {closed }}\left(H_{\kappa^{+}} \cup\left\{\kappa^{+}\right\}\right)^{5}$ holds, and $\kappa^{<\kappa}=\kappa$. Then there is a $\kappa^{+}$-sequence of rigid, pairwise non-isomorphic $\kappa^{+}$-Souslin trees which is able to realize every equivalence relation on $\kappa^{+}$. So $(\star)_{\kappa^{+}}$holds.

Proof. I shall show first that there is such a sequence of rigid, pairwise nonisomorphic $\kappa^{+}$-Souslin trees which is able to realize every simple equivalence relation (this is enough to conclude that $(\star)_{\kappa^{+}}$holds).

Fix a sequence $\vec{T}$ as above. Given an equivalence relation $E$ on $\kappa^{+}$, for $\alpha<\kappa^{+}$, let $\mu_{\alpha}$ be the least member of $[\alpha]_{E}$. Define the forcing notion $\mathbb{P}_{E}$ as follows: Conditions in $\mathbb{P}_{E}$ are of the form $\left\langle p_{\alpha} \mid \alpha<\lambda_{p}\right\rangle$, where $\lambda_{p}<\kappa^{+}$and for every $\alpha<\lambda_{p}$, if $\mu_{\alpha}<\alpha$, then there is some $\gamma<\kappa^{+}$such that $p_{\alpha}$ is an isomorphism between $T^{\mu_{\alpha}} \mid \gamma+1$ and $T^{\alpha} \mid \gamma+1$, the restrictions of these trees to levels less than or equal to $\gamma$. Otherwise, $p_{\alpha}=\emptyset$. The ordering is the obvious one.

The first step now is to check that the statement " $\vec{T}$ is a $\kappa^{+}$-sequence of rigid, non-isomorphic $\kappa^{+}$-Souslin trees which is able to realize every simple equivalence relation on $\kappa^{+}$, as witnessed by $\mathbb{P}_{E}$ ", if true, is $<\kappa^{+}$-closed-necessary.

\footnotetext{
${ }^{5}$ Note that this is the lightface closed maximality principle at $\kappa^{+}$.
} 
The point is that this statement is $\Pi_{1}^{1}\left(H_{\kappa^{+}}\right)$, and hence is preserved under $<\kappa^{+}$-closed forcings. For the quantification over all simple equivalence relations on $\kappa^{+}$is essentially a first order quantification. And the partial order $\mathbb{P}_{E}$ is a subset of $H_{\kappa^{+}}$which is easily definable from $E$.

The next step is to show that the statement can be forced to be true by a $<\kappa^{+}$-closed forcing. This was done in [HT00], assuming that the ground model satisfies $\kappa^{<\kappa}=\kappa$ and $2^{\kappa}=\kappa^{+}$. The former was explicitly demanded, and the latter follows from $\mathrm{MP}_{<\kappa^{+}-\text {closed }}\left(\left\{\kappa^{+}\right\}\right)$by Theorem 3.1 - it even implies $\nabla_{\kappa^{+}}$.

So it is $<\kappa^{+}$-closed-forceably necessary, and hence true.

The restriction to simple equivalence relations can be dropped, but by a proof that necessitates repeating the proof of [HT00]. The argument works as follows: First force with the $<\kappa^{+}$-closed partial order $\mathbb{Q}$ to add the sequence $\vec{T}$ of Souslin trees. $\mathbb{Q}$ consists of conditions $q=\left\langle t_{\alpha}^{q} \mid \alpha<\kappa^{+}\right\rangle$such that for all but $\kappa$ many $\alpha, t_{\alpha}^{q}=\emptyset$, and for all $\alpha, t_{\alpha}^{q}$ is an initial segment of the $\alpha^{\text {th }}$ Souslin tree to be added. Again, the ordering is the obvious one - the forcing can be viewed as a product of the Jech partial order to add a Souslin tree.

Now let $\mathbb{P} \in \mathrm{V}[\vec{T}]$ be $<\kappa^{+}$-closed, and let $G$ be $\mathbb{P}$-generic over $\mathrm{V}[\vec{T}]$. It has to be shown that in $\mathrm{V}[\vec{T}][G], \vec{T}$ is a $\kappa^{+}$-sequence of rigid, pairwise non-isomorphic $\kappa^{+}$-Souslin trees which is able to realize every equivalence relation on $\kappa^{+}$.

So let $E \in \mathrm{V}[\vec{T}][G]$ be an equivalence relation on $\kappa^{+}$(in the sense of any of the models around). Let $H$ be $\mathbb{P}_{E}$-generic over $\mathrm{V}[\vec{T}][G]$. Now $\mathrm{V}[\vec{T}][G][H]$ can be viewed as a one-step extension by $\mathbb{P}_{\vec{T}} * \dot{\mathbb{P}} * \mathbb{P}_{\dot{E}}$, where $\dot{E}$ is a name for $E$. And since the middle forcing notion is $<\kappa^{+}$-closed, the argument from [HT00] goes through with minor changes. The main point is that the following forcing $\mathbb{R} \subseteq \mathbb{Q} * \dot{\mathbb{P}} * \mathbb{P}_{\dot{E}}$ is dense. $\mathbb{R}$ consists of conditions $\langle q, \dot{p}, \check{r}\rangle$, such that, for some $\gamma$ and $\delta<\kappa^{+}$,

1. $\langle q, \dot{p}\rangle$ decides $\dot{E}\lceil\gamma \times \gamma$,

2. for all $\alpha<\gamma, t_{\alpha}^{q}$ has height $\delta+1$, and

3. (a) for all $\alpha<\gamma$, if $\langle q, \dot{p}\rangle \Vdash \mu_{\alpha}<\alpha$, then $r_{\alpha}$ is an isomorphism between $t_{\mu_{\alpha}}^{q}$ and $t_{\alpha}^{q}$, and

(b) $r_{\alpha}=\emptyset$, otherwise.

So $\mathbb{R}$ is forcing equivalent to $\mathbb{Q} * \dot{\mathbb{P}} * \mathbb{P}_{\dot{E}} \cdot \mathbb{R}$ is $<\kappa^{+}$-closed, so it adds no $\kappa$ sequences of ordinals over $\mathrm{V}$, and so, $\mathbb{P}_{E}$ adds no new $\kappa$-sequences of ordinals over $\mathrm{V}[\vec{T}][G]$. Since $\mathbb{P}_{E}$ has size $\kappa^{+}$in $\mathrm{V}[\vec{T}][G]$, it preserves cofinalities as well.

It's obvious that $\mathbb{P}_{E}$ adds isomorphisms between the trees that are to be made isomorphic. What's left to show is that no unwanted isomorphisms are added, and in particular, that the rigidity of the trees is preserved. That argument works as in [HT00], modulo the changes I sketched: One now has to work with $\mathbb{Q} * \dot{\mathbb{P}}$ instead of $\mathbb{Q}$. 


\subsection{Impossible strengthenings of $\mathrm{MP}_{<\kappa-\text { closed }}$}

First, I would like to remark that $\mathrm{MP}_{<\kappa-\text { closed }}$ cannot be consistently strengthened by allowing for parameters which are not in $H_{\kappa^{+}}$. This is simply because for any set $a$, it is $<\kappa$-closed forceably necessary that $a \in H_{\kappa^{+}}$: Just forcing with $\operatorname{Col}(\kappa, \mathrm{TC}(a))$ yields a model which thinks that $a \in H_{\kappa^{+}}$, and this remains true in every further forcing extension. Thus, if $\mathrm{MP}_{<\kappa-\text { closed }}(\{a\})$ is true, then it follows that $a \in H_{\kappa^{+}}$. So in formulating $\mathrm{MP}_{<\kappa-\operatorname{closed}}\left(H_{\kappa^{+}}\right)$, the class of parameters I allowed for was already as large as consistently possible.

It also follows from the observations of the previous section that the necessary version of $\mathrm{MP}_{<\kappa-\text { closed }}\left(H_{\kappa^{+}}\right)$is inconsistent. This was observed jointly by Hamkins and the author for the case $\kappa=\omega_{1}$. Namely, let $\square \mathrm{MP}<\kappa-\operatorname{closed}\left(H_{\kappa^{+}}\right)$ be the principle stating that $\mathrm{MP}_{<\kappa-\text { closed }}\left(H_{\kappa^{+}}\right)$holds in every forcing extension obtained by $<\kappa$-closed forcing (with $H_{\kappa^{+}}$interpreted in the extension).

Theorem 3.16 (Fuchs/Hamkins for $\left.\kappa=\omega_{1}\right) \quad \square \mathrm{MP}<\kappa-$ closed $\left(H_{\kappa^{+}}\right)$is inconsistent with ZFC, if $\kappa>\omega{ }^{6}$

Proof. Assume ZFC $+\square \mathrm{MP}_{<\kappa-\text { closed }}\left(H_{\kappa^{+}}\right)$. Then there is a generic extension obtained by forcing with a $<\kappa$-closed poset, in which there is a slim $\kappa$-Kurepa tree. In this extension, $\mathrm{MP}_{<\kappa-\text { closed }}\left(H_{\kappa^{+}}\right)$has to hold. But this contradicts Theorem 3.3.

Note that it is not the case that the stronger a principle is, the stronger its necessary form is! This is because the meaning of the modal operator $\square$ changes accordingly. So, for example, $\square \mathrm{MP}_{\mathrm{Col}(\kappa)}\left(H_{\kappa^{+}}\right)$means that $\mathrm{MP}_{\mathrm{Col}(\kappa)}\left(H_{\kappa^{+}}\right)$holds in any extension obtained by forcing with a partial order in $\operatorname{Col}(\kappa)$. Indeed, the following questions arise:

\section{Question 3.17}

1. Is $\square \mathrm{MP}_{<\kappa-\text { dir. cl. }}\left(H_{\kappa^{+}}\right)$consistent?

2. Is $\square \mathrm{MP}_{\mathrm{Col}(\kappa)}\left(H_{\kappa^{+}}\right)$consistent?

The issue about the question concerning $\square \mathrm{MP}_{<\kappa-\text { dir. cl. }}\left(H_{\kappa^{+}}\right)$is that it is not possible in general to add a slim $\kappa$-Kurepa tree by $<\kappa$-directed-closed forcing. This is because the existence of a slim $\kappa$-Kurepa tree is incompatible with $\kappa$ being ineffable, but $\kappa$ 's ineffability, and even its supercompactness, can be indestructible under $<\kappa$-directed-closed forcing. However, if $\kappa$ is a successor cardinal, then the standard forcing to add a Kurepa tree (see [Jec03]) is indeed $<\kappa$-directed-closed. So the argument from the proof of Lemma 3.16 gives a partial negative answer to the first question:

Lemma $3.18 \square \mathrm{MP}_{<\kappa-\text { dir. cl. }}\left(H_{\kappa^{+}}\right)$cannot hold at a successor cardinal $\kappa$.

\footnotetext{
${ }^{6}$ In contrast, $\square \mathrm{MP}\left(H_{\omega_{1}}\right)$, i.e., the case where $\kappa=\omega$, is consistent, assuming the consistency of ZF $+\mathrm{AD}_{\mathbb{R}}+$ " $\theta$ is regular". This was shown by Woodin in unpublished work.
} 
Note that since the closed lightface maximality principles are necessary in the appropriate sense by Observation 4.1, their necessary forms do not constitute strengthenings at all.

\section{Separating the principles}

In this section I am turning to the questions about the reversibility of the implications established between the maximality principles. For this, it will be necessary to know that some of the maximality principles are preserved by certain forcings.

Concerning the lightface principles, I would like to make the following observation, analogues of which were made by Hamkins in [Ham03b, Observation 4]. There is also a general account of the phenomenon in [Lei04, Lemma 1.9].

Observation 4.1 $\mathrm{MP}_{<\kappa-\text { closed }}\left(H_{\kappa} \cup\{\kappa\}\right)$, if true, is $<\kappa$-closed-necessary. Actually, $\mathrm{MP}_{<\kappa \text {-closed }}(\{a\})$ persists to $<\kappa$-closed extensions, for any a.

The analogous statements apply to the maximality principles for $<\kappa$-directedclosed forcings and forcings from $\mathrm{Col}(\kappa)$ as well.

Proof. Assume $\mathrm{MP}_{<\kappa-\text { closed }}\left(H_{\kappa} \cup\{\kappa\}\right)$. Let $G \subseteq \mathbb{P}$ be V-generic, where $\mathbb{P}$ is $<\kappa$-closed. If some statement $\varphi$ (involving parameters from $\mathrm{V}$; the point is that $\mathbb{P}$ adds no new members to $\left.H_{\kappa} !\right)$ is $<\kappa$-closed-forceably necessary over $\mathrm{V}[G]$, then the statement $\varphi^{\prime}$, expressing that $\varphi$ is $<\kappa$-closed-necessary, is $<\kappa$-closedforceably necessary over $\mathrm{V}[G]$. Let $\mathbb{Q} \in \mathrm{V}[G]$ be a $<\kappa$-closed notion of forcing witnessing this. Let $\mathbb{Q}$ be a $\mathbb{P}$-name for $\mathbb{Q}$, and let $p \in G$ force that $\dot{\mathbb{Q}}$ forces $\varphi^{\prime}$ to be $<\kappa$-closed-necessary. Then, letting $\mathbb{P}(\leq p)$ be the restriction of $\mathbb{P}$ to conditions below $p, \mathbb{P}(\leq p) * \dot{\mathbb{Q}}$ is a $<\kappa$-closed notion of forcing which makes $\varphi^{\prime}$ $<\kappa$-closed-necessary. So by $\mathrm{MP}_{<\kappa-\text { closed }}\left(H_{\kappa} \cup\{\kappa\}\right), \varphi^{\prime}$ is true in $\mathrm{V}$. This means that $\varphi$ is $<\kappa$-closed-necessary in V. So $\varphi$ is true in $\mathrm{V}[G]$.

Concerning the boldface versions of the maximality principles for $<\kappa$-closed or $<\kappa$-directed-closed forcing, there is the following Lemma:

Lemma 4.2 Assume $\mathrm{MP}_{<\kappa \text {-closed }}\left(H_{\kappa^{+}}\right)$. Let $\mathbb{P}$ be a $<\kappa$-closed notion of forcing which doesn't add sets to $H_{\kappa^{+}}-$this is the case, for example, if $\mathbb{P}$ is $<\kappa^{+}$-closed. If $G$ is $\mathbb{P}$-generic, then in $\mathrm{V}[G], \mathrm{MP}_{<\kappa-\text { closed }}\left(H_{\kappa^{+}}\right)$continues to hold.

Analogously, if $\mathrm{MP}_{<\kappa-\text { dir. cl. }}\left(H_{\kappa^{+}}\right)$holds and $\mathbb{P}$ is an $H_{\kappa^{+}}$-preserving notion of forcing which is $<\kappa$-directed-closed and $G$ is $\mathbb{P}$-generic, then in $\mathrm{V}[G]$, $\mathrm{MP}_{<\kappa-\text { dir. cl. }}\left(H_{\kappa^{+}}\right)$is still true.

Proof. Let $\varphi[\vec{a}]$ be $<\kappa$-closed-forceably necessary over $\mathrm{V}[G]$, where $\vec{a} \in H_{\kappa^{+}}$. Then it is $<\kappa$-closed-forceably necessary over $\mathrm{V}[G]$ that $\varphi[\vec{a}]$ is $<\kappa$-closednecessary. Let this be witnessed the partial order $\mathbb{Q}=\dot{\mathbb{Q}}^{G}$. Let $p \in G$ force over $\mathrm{V}$ that $\mathbb{Q}$ a $<\kappa$-closed forcing that makes $\varphi[\vec{a}]<\kappa$-closed-necessary. Then $\mathbb{P}(\leq p) * \dot{\mathbb{Q}}$ is a $<\kappa$-closed forcing which makes $\varphi[\vec{a}]$ necessary over V. By $\mathrm{MP}_{<\kappa-\text { closed }}\left(H_{\kappa} \cup\{\kappa\}\right)$ then, $\varphi[\vec{a}]$ is already $<\kappa$-closed-necessary in $\mathrm{V}$, and since 
$\mathrm{V}[G]$ is an extension of $\mathrm{V}$ by a $<\kappa$-closed forcing, this means that $\varphi[\vec{a}]$ holds in $\mathrm{V}[G]$.

Note that there is no version of the previous lemma that applies to $\operatorname{Col}(\kappa)$ and $\operatorname{Col}\left(\kappa^{+}\right)$. This has to do with the fact that any nontrivial forcing in $\operatorname{Col}(\kappa)$ adds sets to $H_{\kappa^{+}}$. I will return to this point in section 6 .

I am now ready to separate $\mathrm{MP}_{<\kappa-\text { closed }}\left(H_{\kappa^{+}}\right)$from $\mathrm{MP}_{<\kappa-\operatorname{dir} . \text { cl. }}\left(H_{\kappa} \cup\{\kappa\}\right)$.

Lemma 4.3 Assuming $\kappa$ is supercompact, $\kappa<\delta$ and $\mathrm{V}_{\delta} \prec \mathrm{V}$, there is a forcing extension in which $\mathrm{MP}_{<\kappa-\text { closed }}\left(H_{\kappa} \cup\{\kappa\}\right)$ holds, but $\mathrm{MP}_{<\kappa-\operatorname{dir} \text {. cl. }}\left(H_{\kappa} \cup\{\kappa\}\right)$ does not.

If in addition $\delta$ is inaccessible, then there is a forcing extension in which $\mathrm{MP}_{<\kappa-\text { closed }}\left(H_{\kappa^{+}}\right)$holds, but $\mathrm{MP}_{<\kappa-\text { dir. cl. }}\left(H_{\kappa} \cup\{\kappa\}\right)$ does not.

Proof. The construction is the same in both cases. Let's assume that $\delta$ is inaccessible, so that the boldface result has to be demonstrated. First force with the Laver preparation from [Lav78] to make $\kappa$ 's supercompactness indestructible by $<\kappa$-directed-closed forcings. Then force with $\operatorname{Col}(\kappa,<\delta)$ to make $\mathrm{MP}_{<\kappa-\text { dir. cl. }}\left(H_{\kappa^{+}}\right)$true. Call the resulting model $M$. Since the latter forcing is $<\kappa$-directed-closed, $\kappa$ is supercompact in $M$, and its supercompactness is indestructible over $M$ by $<\kappa$-directed-closed forcings. Now let $G$ be generic over $M$ for a forcing $\mathbb{P}$ which is $<\kappa^{+^{M}}$-closed and destroys $\kappa$ 's supercompactness (such $\mathbb{P}$ exists, by $[\mathrm{KY} 06]$. It adds a $\kappa^{+}$-regressive $\kappa^{+}$-Kurepa tree). Let $N=M[G]$. $M$ is a model of $\mathrm{MP}_{<\kappa-\text { dir. cl. }}\left(H_{\kappa^{+}}\right)$, hence also a model of $\mathrm{MP}_{<\kappa-\text { closed }}\left(H_{\kappa^{+}}\right)$, and $N$ is a forcing extension of $M$ by a $<\kappa^{+M}$-closed forcing. So $N$ is also a model of $\mathrm{MP}_{<\kappa-\operatorname{closed}}\left(H_{\kappa^{+}}\right)$, by Lemma 4.2. But I claim that $N$ is not a model of $\mathrm{MP}_{<\kappa-\text { dir. cl. }}\left(H_{\kappa} \cup\{\kappa\}\right)$. To see this: Working in $N$, pick $\theta \geq \overline{\overline{\mathbb{P}}}$ such that $\theta^{<\kappa}=\theta$, and then force with $\operatorname{Col}(\kappa, \theta)$ over $N$. Note that $\operatorname{Col}(\kappa, \theta)$ is absolute between $N$ and $M$. Since $\mathbb{P} \times \operatorname{Col}(\kappa, \theta)$ has size $\theta$, is $<\kappa$-closed and collapses $\theta$, some dense subset of it is isomorphic to $\operatorname{Col}(\kappa, \theta)$. But the latter forcing is $<\kappa$-directed-closed, and so the extension $N^{\operatorname{Col}(\kappa, \theta)}=M^{\mathbb{P} \times \operatorname{Col}(\kappa, \theta)}$ can be viewed as $M^{\operatorname{Col}(\kappa, \theta)}$, i.e., as a forcing extension of $M$ by a $<\kappa$-directed-closed forcing. So $\kappa$ is $<\kappa$-directed-closed-necessarily supercompact in $N^{\mathrm{Col}(\kappa, \theta)}$, and hence it is $<\kappa$-directed-closed-forceably necessarily supercompact over $N$. But it is not supercompact in $N$, which shows that $\mathrm{MP}_{<\kappa-\text { dir. cl. }}\left(H_{\kappa} \cup\{\kappa\}\right)$ fails in $N$.

So this shows that the implications from $\mathrm{MP}_{<\kappa-\text { dir. cl. }}\left(H_{\kappa} \cup\{\kappa\}\right)$ to $\mathrm{MP}_{<\kappa-\text { closed }}\left(H_{\kappa} \cup\{\kappa\}\right)$ and from $\mathrm{MP}_{<\kappa-\text { dir. cl. }}\left(H_{\kappa^{+}}\right)$to $\mathrm{MP}_{<\kappa-\text { closed }}\left(H_{\kappa^{+}}\right)$cannot be reversed, assuming supercompactness of $\kappa$, with a reflecting (inaccessible) $\operatorname{rank} \delta$ above. It is unclear whether this is necessary.

Now, let's turn to the question whether $\mathrm{MP}_{<\kappa-\text { dir. cl. }}\left(H_{\kappa} \cup\{\kappa\}\right)$ implies $\mathrm{MP}_{\mathrm{Col}(\kappa)}\left(H_{\kappa} \cup\{\kappa\}\right)$, and the boldface version thereof.

\section{Lemma 4.4}

1. $\mathrm{MP}_{\mathrm{Col}(\kappa)}(\emptyset)$ implies that $\mathrm{V} \neq \mathrm{HOD}$. 
2. If $\mathrm{V}$ is a countable transitive model of $\mathrm{MP}_{<\kappa-c l o s e d}\left(H_{\kappa} \cup\{\kappa\}\right)$, then there is a forcing extension of $L^{\mathrm{V}}$ in which $\mathrm{MP}_{<\kappa-\text { dir. cl. }}\left(H_{\kappa} \cup\{\kappa\}\right)+\mathrm{V}=\mathrm{HOD}$ holds. Analogously, $\mathrm{MP}_{<\kappa-\text { closed }}\left(H_{\kappa^{+}}\right)$implies that there is a forcing extension of $L$ in which $\mathrm{MP}_{<\kappa-\text { dir. cl. }}\left(H_{\kappa^{+}}\right)+\mathrm{V}=\mathrm{HOD}$ holds.

So in general, $\mathrm{MP}_{<\kappa-\text { dir. cl. }}\left(H_{\kappa^{+}}\right)$does not imply $\mathrm{MP}_{\mathrm{Col}(\kappa)}\left(H_{\kappa} \cup\{\kappa\}\right)$, which answers the above questions in the negative.

Proof. Part 1 follows since the statement "V $\neq$ HOD" is $\mathrm{Col}(\kappa)$-forceably necessary. Namely, if $G$ is V-generic for some nontrivial collapse, then $\mathrm{HOD}^{\mathrm{V}[G]} \subseteq \mathrm{V}$, by homogeneity. So $G \in \mathrm{V}[G] \backslash \mathrm{HOD}^{\mathrm{V}[G]}$. And if $H$ is generic for some further collapse over $\mathrm{V}[G]$, "V $\neq$ HOD" becomes even more true, since $\mathrm{HOD}^{\mathrm{V}[G][H]} \subseteq$ $\mathrm{HOD}^{\mathrm{V}[G]} \subseteq \mathrm{V}$. So $\mathrm{V} \neq \mathrm{HOD}$ is $\operatorname{Col}(\kappa)$-necessary in $\mathrm{V}[G]$, and hence true in $\mathrm{V}$, by $\mathrm{MP}_{\mathrm{Col}(\kappa)}(\emptyset)$.

Let's turn to part 2. In case $\mathrm{MP}_{<\kappa-\text { closed }}\left(H_{\kappa} \cup\{\kappa\}\right)$ is assumed, let $\delta=\kappa^{+}$, and in case only $\mathrm{MP}_{<\kappa-\text { closed }}(\emptyset)$ holds, let $\delta$ be the supremum of the ordinals which are definable over $L$ in the parameter $\kappa$. So in any case, $L_{\delta} \prec L$, and in case the boldface principle was assumed, $\delta$ is regular (and hence inaccessible) in $L$. Now let $G$ be $\operatorname{Col}(\kappa,<\delta)$-generic over $L$. So $\operatorname{MP}_{\operatorname{Col}(\kappa)}\left(H_{\kappa} \cup\{\kappa\}\right)$ holds in $L[G]$; if $\delta$ is inaccessible in $L$, even the boldface version follows. $G$ can be viewed as a set of ordinals. Now force to code $G$ as well as the new generic into the continuum function well above $\kappa$, adapting a technique of [Rei06]. Sketch: Do a length $\omega$ iteration, so that $\mathbb{P}_{0}$ codes $G$ into the continuum function above $\kappa^{+} . \mathbb{P}_{1}$ codes the $\mathbb{P}_{0}$-generic into the continuum function above $\overline{\overline{P_{0}}}$, and so on. The coding can be arranged in such a way that the intervals of the continuum function coding the $n^{\text {th }}$ generic can be read off the continuum function as well. Let $\mathbb{P}_{\omega}$ be the full support limit of this iteration. In $M=L[G]\left[G_{\omega}\right], G$ and $G_{\omega}$ can be read off the continuum function, and hence are OD. Since every member of $M$ is of the form $\tau\left[G * G_{\omega}\right]$, where $\tau \in L$, it follows that $\mathrm{V}=$ HOD holds in $M$. But since the coding took place above $\kappa$, the forcing $\mathbb{P}_{\omega}$ is $<\kappa$-directedclosed. So $M$ is still a model of $\mathrm{MP}_{<\kappa-\text { dir. cl. }}\left(H_{\kappa} \cup\{\kappa\}\right)$, and even a model of $\mathrm{MP}_{<\kappa-\text { dir. cl. }}\left(H_{\kappa^{+}}\right)$, if $L[G]$ was.

For the record, let's draw the conclusion that the boldface principles are strictly stronger than their lightface counterparts.

\section{Lemma 4.5}

1. Assuming $\mathrm{MP}_{<\kappa-\text { closed }}\left(H_{\kappa} \cup\{\kappa\}\right)$, there is a forcing extension in which $\mathrm{MP}_{<\kappa-\text { closed }}\left(H_{\kappa} \cup\{\kappa\}\right)$ holds but $\mathrm{MP}_{<\kappa-\text { closed }}\left(H_{\kappa^{+}}\right)$fails.

2. Assuming $\mathrm{MP}_{<\kappa-\text { closed }}\left(H_{\kappa} \cup\{\kappa\}\right)$ holds in a countable transitive model of ZFC, there is such a model in which $\mathrm{MP}_{<\kappa-\text { dir. cl. }}\left(H_{\kappa} \cup\{\kappa\}\right)$ holds but $\mathrm{MP}_{<\kappa-\operatorname{closed}}\left(H_{\kappa^{+}}\right)$fails.

3. Assuming $\mathrm{MP}_{<\kappa-\text { closed }}\left(H_{\kappa} \cup\{\kappa\}\right)$ holds in a countable transitive model of ZFC, there is such a model in which $\mathrm{MP}_{\mathrm{Col}(\kappa)}\left(H_{\kappa} \cup\{\kappa\}\right)$ holds but $\mathrm{MP}_{<\kappa-\text { closed }}\left(H_{\kappa^{+}}\right)$fails. 
Proof. The first point can be proved by adding a slim $\kappa$-Kurepa tree over a model of $\mathrm{MP}_{<\kappa \text {-closed }}\left(H_{\kappa} \cup\{\kappa\}\right)$ with $<\kappa$-closed forcing, which preserves the particular lightface principle, according to Observation 4.1. In the resulting model, the boldface version of the principle fails, since that would imply that there is no slim $\kappa$-Kurepa tree, by Theorem 3.3.

Since the second statement is weaker than the third, it suffices to prove the latter. Since it is clear by now how to obtain a transitive model in which $\mathrm{MP}_{\mathrm{Col}(\kappa)}\left(H_{\kappa} \cup\{\kappa\}\right)$ holds from one in which $\mathrm{MP}_{<\kappa-\operatorname{closed}}\left(H_{\kappa} \cup\{\kappa\}\right)$ holds (by collapsing the supremum of all ordinals which are $L$-definable in $\kappa$ to $\kappa$ over $L$ ), we may assume that $\mathrm{MP}_{\mathrm{Col}(\kappa)}\left(H_{\kappa} \cup\{\kappa\}\right)$ holds. If $\mathrm{MP}_{<\kappa-\operatorname{closed}}\left(H_{\kappa^{+}}\right)$fails, then there is nothing left to show, so assume that $\mathrm{MP}_{<\kappa-\text { closed }}\left(H_{\kappa^{+}}\right)$holds. Letting $\delta=\kappa^{+}$, it follows that $L_{\delta} \prec L$ and $\delta$ is inaccessible in $L$. So the set $R$ of $\mu<\delta$ with $L_{\mu} \prec L_{\delta} \prec L$ is club in $\delta$. Let $\delta_{0}$ and $\delta_{1}$ be the least two ordinals in that set which are greater than $\kappa$. Now let $M=L_{\delta_{1}}$, and force with $\operatorname{Col}\left(\kappa,<\delta_{0}\right)$ over $M$ to produce a model $M[G]$. In $M[G]$, the principle $\mathrm{MP}_{\mathrm{Col}(\kappa)}\left(H_{\kappa} \cup\{\kappa\}\right)$ holds, while $\mathrm{MP}_{<\kappa-\text { closed }}\left(H_{\kappa^{+}}\right)$fails. For otherwise, letting $\delta^{\prime}=\left(\kappa^{+}\right)^{M[G]}$, it would follow that $L_{\delta^{\prime}} \prec L^{M[G]}=L_{\delta_{1}} \prec L_{\delta}$, so that $\delta_{0} \leq \delta^{\prime}<\delta_{1}$ and $\delta^{\prime} \in R$. This means that $\delta_{0}=\delta^{\prime}$, by the choice of $\delta_{0}$ and $\delta_{1}$. But then, since $\delta^{\prime}$ is regular in $M[G]$, it is inaccessible in $L^{M[G]}=L_{\delta_{1}} \prec L$, and hence in $L$. So $\delta^{\prime}$ is a limit point of $R$, since $\delta^{\prime}=\delta_{0}$. But then it can't be the least member of $R$ above $\kappa$. $\square$

So in general, none of the implications shown in Figure 1 can be reversed, as is shown by Lemmas 4.3, 4.4 and 4.5 .

Since it fits in here, I would now like to prove the following preservation lemma for $\mathrm{MP}_{\mathrm{Col}(\kappa)}\left(H_{\kappa^{+}}\right)$:

Lemma 4.6 Assume $\mathrm{MP}_{\mathrm{Col}(\kappa)}\left(H_{\kappa^{+}}\right)$, and let $\mathbb{P}$ be a $<\kappa$-closed notion of forcing which is an element of $H_{\kappa^{+}}$. Let $G \subseteq \mathbb{P}$ be $\mathrm{V}$-generic for $\mathbb{P}$. Then in $\mathrm{V}[G]$, $\mathrm{MP}_{\mathrm{Col}(\kappa)}\left(H_{\kappa^{+}}\right)$continues to hold. ${ }^{7}$

Proof. Let $\mathbb{P}$ and $G$ be as in the statement of the lemma. Let $\varphi(a)$ be $\operatorname{Col}(\kappa)$ forceably necessary in $\mathrm{V}[G]$, where $a$ is a subset of $\kappa$ (every member of $H_{\kappa^{+}}$can be so coded).

Let $\operatorname{Col}(\kappa, \delta)$ force over $\mathrm{V}[G]$ that $\varphi(a)$ is $\operatorname{Col}(\kappa)$-necessary. I can assume that $\delta \geq \overline{\overline{\mathbb{P}}}$ and $\delta^{<\kappa}=\delta$ (in $\mathrm{V}[G]$ ). Let $H$ be $\operatorname{Col}(\kappa, \delta)$-generic over $\mathrm{V}[G]$, so that in $\mathrm{V}[G][H], \varphi(a)$ is $\operatorname{Col}(\kappa)$-necessary.

Pick a $\mathbb{P}$-name $\dot{a} \in H_{\kappa^{+}}$for a (e.g., a nice $\mathbb{P}$-name for a subset of $\check{\kappa}$ ). Since $\mathrm{V}[G][H]=\mathrm{V}[H][G]$, there is some $p \in G$ such that

$(*) \mathrm{V}[H] \models\left(p \Vdash_{\mathbb{P}} \varphi(\dot{a})\right.$ is $\operatorname{Col}(\kappa)$-necessary $)$.

Claim: The statement " $p \Vdash_{\mathbb{P}} \varphi(\dot{a})$ " is $\operatorname{Col}(\kappa)$-necessary in $\mathrm{V}[H]$.

Proof of Claim. Let $I$ be $\operatorname{Col}(\kappa, \mu)$-generic over $\mathrm{V}[H]$, for some $\mu$. I have to show that $\mathrm{V}[H][I]\left[G^{\prime}\right] \models \varphi\left(\dot{a}^{G^{\prime}}\right)$, whenever $G^{\prime} \ni p$ is $\mathbb{P}$-generic over $\mathrm{V}[H][I]$.

\footnotetext{
${ }^{7}$ There is essentially only one such forcing, namely the forcing $\operatorname{Add}(\kappa)$ to add a subset to $\kappa$, as was pointed out by the referee.
} 
But $\mathrm{V}[H][I]\left[G^{\prime}\right]=\mathrm{V}[H]\left[G^{\prime}\right][I]$. By $(*), \varphi\left(\dot{a}^{G^{\prime}}\right)$ is $\mathrm{Col}(\kappa)$-necessary in $\mathrm{V}[H]\left[G^{\prime}\right]$, and thus true in $\mathrm{V}[H]\left[G^{\prime}\right][I]$, as claimed.

And that's it: $\mathrm{By} \mathrm{MP}_{\mathrm{Col}(\kappa)}\left(H_{\kappa^{+}}\right)$, it follows from the claim that $p \Vdash_{\mathbb{P}} \varphi(\dot{a})$. So $\varphi(a)$ is true in $\mathrm{V}[G]$.

For $\mathrm{MP}_{<\kappa-\text { closed }}\left(H_{\kappa^{+}}\right)$, more is true. First, note the following fact, which is not hard to verify. One direction has already tacitly been made use of at many places in this paper, and now the converse will be needed.

Fact 4.7 Let $\kappa$ be regular, $\mathbb{P}$ a notion of forcing and $\dot{\mathbb{Q}}$ a $\mathbb{P}$-name such that $\mathbb{P} \Vdash \dot{\mathbb{Q}}$ is a notion of forcing. Then the following statements are equivalent:

1. $\mathbb{P}$ is $<\kappa$-closed and $\Vdash_{\mathbb{P}} \dot{\mathbb{Q}}$ is $<\kappa$-closed.

2. $\mathbb{P} * \dot{\mathbb{Q}}$ is $<\kappa$-closed.

I shall need the technique of term forcing (or termspace forcing). Given a forcing $\mathbb{P} * \dot{\mathbb{Q}}$, the term forcing of $\mathbb{Q}$ over $\mathbb{P}$ (as it is referred to in [HW05]), which I shall denote by $\mathcal{A}(\mathbb{P}, \mathbb{Q})$ (as in $[\mathrm{FK} 03]$ ), consists of $\mathbb{P}$-names $\tau$ such that $\Vdash_{\mathbb{P}}(\tau \in \dot{\mathbb{Q}})$. One can work with a full set of such names, by picking one representative out of every equivalence class consisting of names which are forced to be equal. The ordering is the obvious one: $\tau_{1} \leq \tau_{2}$ iff $\vdash_{\mathbb{P}}\left(\tau_{1} \leq_{\dot{\mathbb{Q}}} \tau_{2}\right)$. The following is the fundamental Lemma on term forcing.

Lemma 4.8 Let $\mathbb{P}$ be a p.o., $\mathbb{P} \Vdash \mathbb{Q}$ is a p.o., and let $\mathcal{A}=\mathcal{A}(\mathbb{P}, \dot{\mathbb{Q}})$. Suppose that I is $\mathcal{A}$-generic over $\mathrm{V}$, and suppose that $\mathrm{V}^{\prime} \supset \mathrm{V}[I]$ is a transitive model of ZFC. If there is a $G \in \mathrm{V}^{\prime}$ which is $\mathbb{P}$-generic over $\mathrm{V}$, then $\mathrm{V}^{\prime}$ also contains a filter $H$ which is $\dot{\mathbb{Q}}_{G}$-generic over $\mathrm{V}[G]$.

Proof. One lets $H=\left\{\tau_{G} \mid \tau \in I\right\}$. A proof that this works can be found in [HW05].

I shall also need the following fact, which is again easily verified:

Fact 4.9 If $\kappa$ is a regular cardinal such that $\mathbb{P}$ is $<\kappa$-closed and $\mathbb{P} \Vdash \dot{\mathbb{Q}}$ is $<\kappa$-closed, then $\mathcal{A}(\mathbb{P}, \dot{\mathbb{Q}})$ is $<\kappa$-closed.

Lemma 4.10 Assume $\mathrm{MP}_{<\kappa-\text { closed }}\left(H_{\kappa^{+}}\right)$. Let $\mathbb{P}=\prod_{i \in I} \mathbb{P}_{i}$, with $<\kappa$ support, where each $\mathbb{P}_{i}$ is a $<\kappa$-closed forcing which is an element of $H_{\kappa^{+}}$. Then if $G$ is $\mathbb{P}$-generic over $\mathrm{V}, \mathrm{V}[G]$ still satisfies $\mathrm{MP}_{<\kappa-\text { closed }}\left(H_{\kappa^{+}}\right)$.

Proof. The method of the proof of [HW05, Thm. 10] applies.

So let $\mathbb{P}$ and $G$ be as in the statement of the lemma. Let $\varphi(a)$ be $<\kappa$-closedforceably necessary in $\mathrm{V}[G]$, where $a$ is a subset of $\kappa$ (every member of $H_{\kappa^{+}}$can be so coded). Since $\mathrm{MP}_{<\kappa \text {-closed }}\left(H_{\kappa^{+}}\right)$holds, it follows that $\kappa^{<\kappa}=\kappa$, so that a $\Delta$-system argument shows that $\mathbb{P}$ is $\kappa^{+}$-c.c. This means that there is a set $I_{0} \subseteq I$ of size $\kappa$ such that $a \in \mathrm{V}\left[G_{0}\right]$, where $G$ is viewed as $G_{0} \times G_{1}, G_{0}$ being 
generic for $\mathbb{P}_{0}=\prod_{i \in I_{0}} \mathbb{P}_{i}$ over $\mathrm{V}$ and $G_{1}$ being $\mathbb{P}_{1}=\prod_{i \in I \backslash I_{0}} \mathbb{P}_{i}$-generic over $\mathrm{V}\left[G_{0}\right]$.

By rearranging the indices, one may assume that $I_{0}$ is transitive. So, since

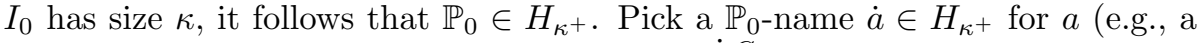
nice $\mathbb{P}_{0}$-name for a subset of $\check{\kappa}$ ), and let $\mathbb{Q}=\dot{\mathbb{Q}}^{G}$ force over $\mathrm{V}[G]$ that $\varphi(\dot{a})$ is $<\kappa$-closed-necessary, where $\mathbb{P} \Vdash \dot{\mathbb{Q}}$ is $<\kappa$-closed. Let $p_{0} \in \mathbb{P}$ force that $\dot{\mathbb{Q}}$ forces that $\varphi(\dot{a})$ is $<\kappa$-closed-necessary. $\mathbb{P}_{0}$ can be picked so that $p_{0} \in \mathbb{P}_{0}$. Then $p_{0}$ forces with respect to $\mathbb{P}_{0}$ that $\mathbb{P}_{1} * \dot{\mathbb{Q}}$ forces that $\varphi(\dot{a})$ is $<\kappa$-closed-necessary.

Claim: The statement " $p_{0} \Vdash_{\mathbb{P}_{0}} \varphi(\dot{a})$ " is $<\kappa$-closed-forceably necessary.

Proof of Claim. Set $\mathcal{A}=\mathcal{A}(\mathbb{P}, \dot{\mathbb{Q}})$. I am going to argue that forcing with $\mathbb{P}_{1} \times \mathcal{A}$ makes the statement in question $<\kappa$-closed-necessary. Knowing this, the claim is proven, since by Fact $4.9, \mathcal{A}$ is $<\kappa$-closed.

So let $G_{1}^{\prime} \times I$ be $\mathbb{P}_{1} \times \mathcal{A}$-generic over $\mathrm{V}$. It has to be shown that if $H^{\prime}$ is generic over $\mathrm{V}\left[G_{1}^{\prime}\right][I]$ for some $<\kappa$-closed forcing and $G_{0}^{\prime}$ is a $\mathbb{P}_{0^{-}}$-generic filter over $\mathrm{V}\left[G_{1}^{\prime}\right][I]\left[H^{\prime}\right]$ such that $p_{0} \in G_{0}^{\prime}$, then $\varphi(a)$ holds in $\mathrm{V}\left[G_{1}^{\prime}\right][I]\left[H^{\prime}\right]\left[G_{0}^{\prime}\right]=$ $\mathrm{V}\left[G^{\prime}\right][I]\left[H^{\prime}\right]$ (letting $G^{\prime}=G_{0}^{\prime} \times G_{1}^{\prime}$ ). In that situation, by the fundamental Lemma 4.8 , there is in $\mathrm{V}\left[G^{\prime}\right][I]$ a filter $H$ which is $\dot{\mathbb{Q}}_{G^{\prime}}$-generic over $\mathrm{V}\left[G^{\prime}\right]$. Note that in $\mathrm{V}\left[G^{\prime}\right][H], \varphi(a)$ is $<\kappa$-closed-necessary, by assumption on $p_{0}$. Now $\mathrm{V}\left[G^{\prime}\right][I]\left[H^{\prime}\right]$ can be viewed as $\mathrm{V}\left[G^{\prime}\right][H][J]\left[H^{\prime}\right]$, where $J$ is $\mathbb{C}=\mathcal{A} /\left(\dot{\mathbb{Q}}_{G^{\prime}}\right)$ generic over $\mathrm{V}\left[G^{\prime}\right][H]$, so that $\mathrm{V}\left[G^{\prime}\right][H][J]=\mathrm{V}[G][I]$. Let $\dot{\mathbb{C}}$ be a name for $\mathbb{C}$. In $\mathrm{V}\left[G^{\prime}\right], \mathcal{A} \cong \mathbb{Q}_{G^{\prime}} * \dot{\mathbb{C}}$ is $<\kappa$-closed, and $\dot{\mathbb{Q}}_{G^{\prime}}$ is $<\kappa$-closed, so by Lemma 4.7 , it follows that (in $\mathrm{V}\left[G^{\prime}\right]$ ) $\dot{\mathbb{Q}}_{G} \Vdash \dot{\mathbb{C}}$ is $<\kappa$-closed. So, it follows that $\mathrm{V}\left[G^{\prime}\right][I]\left[H^{\prime}\right]=\mathrm{V}\left[G^{\prime}\right][H][J]\left[H^{\prime}\right]$ is a $<\kappa$-closed forcing extension of $\mathrm{V}\left[G^{\prime}\right][H]$, where $\varphi(a)$ is $<\kappa$-closed-necessary. So $\varphi(a)$ is true in $\mathrm{V}\left[G^{\prime}\right][I][H]$, as was to be shown.

$\square_{\text {Claim }}$

Now the proof can be completed: By $\mathrm{MP}_{<\kappa-\text { closed }}\left(H_{\kappa^{+}}\right)$, it follows from the claim that the statement " $p_{0} \Vdash \varphi(\dot{a})$ " is not only true but $<\kappa$-closed-necessary in V. So it is true in $\mathrm{V}\left[G_{1}\right]$. Since $p_{0} \in G_{0}$, it follows that $\varphi$ is true of $a=\dot{a}_{G_{0}}$ in $\mathrm{V}\left[G_{1}\right]\left[G_{0}\right]=\mathrm{V}[G]$.

Now the question about whether or not $\mathrm{MP}_{<\kappa-\text { closed }}\left(H_{\kappa^{+}}\right)$implies that $2^{\kappa}=$ $\kappa^{+}$can be answered, in the negative.

Corollary 4.11 MP $<_{\kappa-\text { closed }}\left(H_{\kappa^{+}}\right)$does not determine the value of $2^{\kappa}$.

Proof. Forcing to add as many Cohen subsets of $\kappa$ as wished preserves the principle $\mathrm{MP}_{<\kappa-\text { closed }}\left(H_{\kappa^{+}}\right)$, by the previous Lemma 4.10.

It is not hard to see that even $\mathrm{MP}_{<\kappa-\text { dir. cl. }}\left(H_{\kappa^{+}}\right)$does not imply that $2^{\kappa}=\kappa^{+}$, by adapting an argument from [Ham03b, Theorem 12] to the present context.

Lemma 4.12 $\mathrm{MP}_{<\kappa-\text { dir. cl. }}\left(H_{\kappa^{+}}\right)$does not determine the value of $2^{\kappa}$. 
Proof. Starting in a universe in which $\mathrm{MP}_{<\kappa-\text { dir. cl. }}\left(H_{\kappa^{+}}\right)$, it has been shown already that there is an inner model (which I shall refer to as $\mathrm{V}$ ) in which $\kappa<\delta$ and $\mathrm{V}_{\delta} \prec \mathrm{V}$ holds. Let $G$ be $\operatorname{Col}(\kappa,<\delta)$-generic over $\mathrm{V}$, so that $\mathrm{MP}_{<\kappa-\text { dir. cl. }}\left(H_{\kappa^{+}}\right)$ holds in $\mathrm{V}[G]$. I claim that forcing to add as many Cohen subsets of $\kappa$ over $\mathrm{V}[G]$ as desired, preserves $\mathrm{MP}_{<\kappa-\text { dir. cl. }}\left(H_{\kappa^{+}}\right)$.

So let $H$ be generic over $\mathrm{V}[G]$ for the $\lambda$-fold product of Cohen forcing to add a subset of $\kappa, \operatorname{Add}(\kappa)$, with $<\kappa$ support. Let $a \subseteq \kappa \in \mathrm{V}[G][H]$, so that $\varphi(a)$ is $<\kappa$-directed-closed-forceably necessary over $\mathrm{V}[G][H]$. Then $a$ is already added by some product of $\operatorname{Add}(\kappa)$ 's of size less than $\kappa$, by the $\kappa$-c.c. So one may arrange things so that $a \in \mathrm{V}[G]\left[H_{0}\right]$, where $H_{0}$ is generic for $\mathbb{P}_{0}:=\operatorname{Add}(\kappa)^{\gamma}$ over $\mathrm{V}[G]$, where $\gamma<\kappa$, and $H_{1}$ is $\mathbb{P}_{1}:=\operatorname{Add}(\kappa)^{\lambda-\gamma}$-generic over $\mathrm{V}[G]\left[H_{0}\right]$ so that $\mathrm{V}[G]\left[H_{0}\right]\left[H_{1}\right]=\mathrm{V}[G][H]$.

Now the order of extension can be reversed, so that $\mathrm{V}[G][H]=\mathrm{V}\left[H_{0}\right][G]\left[H_{1}\right]$. Since $\mathbb{P}_{0} \in \mathrm{V}_{\delta}$, it follows that $V_{\delta}^{\mathrm{V}\left[H_{0}\right]} \prec \mathrm{V}\left[H_{0}\right]$ and $\delta$ is inaccessible in $\mathrm{V}\left[H_{0}\right]$. So in $\mathrm{V}\left[H_{0}\right][G], \mathrm{MP}_{<\kappa-\text { dir. cl. }}\left(H_{\kappa^{+}}\right)$holds, by Theorem 2.10. So in particular, $\mathrm{MP}_{<\kappa-\text { dir. cl. }}(\{a\})$ holds there. By Lemma 4.1, it persists to $\mathrm{V}[G][H]$. Since $a$ was an arbitrary subset of $\kappa$ in $\mathrm{V}[G][H], \mathrm{MP}_{<\kappa-\text { dir. cl. }}\left(H_{\kappa^{+}}\right)$holds in $\mathrm{V}[G][H]$. open.

But the question whether $\mathrm{MP}_{\mathrm{Col}(\kappa)}\left(H_{\kappa^{+}}\right)$implies that $2^{\kappa}=\kappa^{+}$remains

I will close this section with some observations on effects of maximality principles on HOD-like models.

Observation 4.13 Assuming $\mathrm{MP}_{\mathrm{Col}(\kappa)}\left(H_{\kappa^{+}}\right), \kappa^{+}$is inaccessible in $\mathrm{HOD}_{A}$, for any $A \subseteq \kappa$.

Proof. Let $\alpha<\kappa^{+}$be given. Then the statement "P $(\alpha)$ HOD $_{A}$ has cardinality $\kappa$ " is $\operatorname{Col}(\kappa)$-forceably necessary. This is because it is necessary after forcing with $\operatorname{Col}\left(\kappa, \overline{\overline{\mathcal{P}(\alpha) \cap \mathrm{HOD}_{A}}}\right)$ - the further one collapses, the smaller the $\mathrm{HOD}_{A} \mathrm{~s}$ get. So the statement is true, by $\mathrm{MP}_{\mathrm{Col}(\kappa)}\left(H_{\kappa^{+}}\right)$.

Observation 4.14 Assuming $\mathrm{MP}_{\mathrm{Col}(\kappa)}\left(H_{\kappa^{+}}\right), \mathrm{HOD}_{A} \cap \mathcal{P}(\kappa)$ is stable, for any $A \subseteq \kappa$. I.e., if $G$ is $\mathrm{V}$-generic for $\operatorname{Col}(\kappa, \lambda)$, then $\operatorname{HOD}_{A}^{\mathrm{V}}{ }^{[G]} \cap \mathcal{P}(\kappa)=\operatorname{HOD}_{A}^{\mathrm{V}} \cap$ $\mathcal{P}(\kappa)$.

Proof. The inclusion from left to right is clear. Now suppose $B \subseteq \kappa$ is in $\operatorname{HOD}_{A}^{\mathrm{V}}$

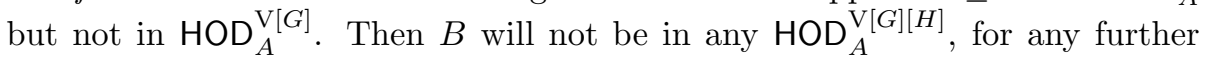
$\operatorname{Col}\left(\kappa, \lambda^{\prime}\right)$-generic $H$. So $B$ is forceably necessarily not in $\operatorname{HOD}_{A}$, and this means that $B \notin \mathrm{HOD}_{A}$, a contradiction.

Assuming $\mathrm{MP}_{<\kappa-\text { closed }}\left(H_{\kappa} \cup\{\kappa\}\right)$, there is another "canonical inner model" that's invariant under forcings in $\operatorname{Col}(\kappa)$. Namely, let

$$
\lim _{\kappa} \mathrm{HOD}=\bigcap_{\lambda>\kappa} \mathrm{HOD}^{\mathrm{V}^{\mathrm{Col}(\kappa, \lambda)} .}
$$


So $a \in \lim _{\kappa}$ HOD iff for every $\lambda>\kappa, \mathbb{1} \Vdash_{\mathrm{Col}(\kappa, \lambda)} \check{a} \in \mathrm{HOD}$. Clearly, if $G$ is generic for some $<\kappa$-closed notion of forcing $\mathbb{P}$, then $\lim _{\kappa} \mathrm{HOD}^{\mathrm{V}}=\lim _{\kappa} \mathrm{HOD}^{\mathrm{V}[G]}$, as $G$ can be absorbed by a collapse to $\kappa$.

It is easy to see that $\lim _{\kappa}$ HOD is an inner model of ZFC: First note that for any $\alpha$, there is a least ordinal $\lambda_{\alpha}$ such that $\mathrm{V}_{\alpha} \cap \lim _{\kappa} \mathrm{HOD}=\mathrm{V}_{\alpha} \cap \mathrm{HOD}^{\mathrm{Col}\left(\kappa, \lambda_{\alpha}\right)}=$ $\mathrm{V}_{\alpha}^{\mathrm{HOD}}{ }^{\operatorname{Col}\left(\kappa, \lambda_{\alpha}\right)}$. So $\lim _{\kappa} \mathrm{HOD}=\bigcup_{\alpha} \mathrm{V}_{\alpha} \cap \mathrm{HOD}^{\operatorname{Col}\left(\kappa, \lambda_{\alpha}\right)}$. Now Powerset and Choice are easy to verify. Next, let's turn to Replacement. Let $a \in \lim _{\kappa} \mathrm{HOD}$, and let $F: a \longrightarrow \lim _{\kappa}$ HOD be definable in $\lim _{\kappa}$ HOD. Fix $\lambda>\kappa$ arbitrarily. Then $\lim _{\kappa} \mathrm{HOD}$ is definable in $\mathrm{V}^{\mathrm{Col}(\kappa, \lambda)}$ in the parameter $\kappa$, and so, since $F$ is definable over $\lim _{\kappa} \mathrm{HOD}$ in some parameters $\vec{p}$ which, in turn, are in $\mathrm{HOD}^{\mathrm{Col}(\kappa, \lambda)}$, $F$ is ordinal definable in $\mathrm{V}^{\mathrm{Col}(\kappa, \lambda)}$; one just relativizes the definition of $F$ over $\lim _{\kappa}$ HOD to $\lim _{\kappa}$ HOD, and substitutes for the parameters their ordinal definitions. But $a$ is ordinal definable in $\mathrm{V}^{\mathrm{Col}(\kappa, \lambda)}$ as well, and hence, so is $F^{\prime \prime} a$. Thus, $F^{\prime \prime} a \in \mathrm{HOD}^{\operatorname{Col}(\kappa, \lambda)} . \lambda$ was arbitrary, so $F^{\prime \prime} a \in \lim _{\kappa} \mathrm{HOD}$, which is what had to be shown for Replacement. The argument for Separation is analogous. The remaining axioms of ZFC are unproblematic.

Lemma 4.15 If $\mathrm{MP}_{<\kappa-\text { closed }}\left(H_{\kappa^{+}}\right)$holds, then, letting $\delta=\kappa^{+}$, it follows that $\delta$ is inaccessible in $\lim _{\kappa} \mathrm{HOD}$ and that $\mathrm{V}_{\delta}^{\lim _{\kappa} \mathrm{HOD}} \prec \lim _{\kappa} \mathrm{HOD}$.

Proof. The proof of Theorem 3.8 works.

\section{Combining the principles}

Let's assume there are regular cardinals $\kappa_{0}<\delta_{0} \leq \kappa_{1}<\delta_{1}$ so that $\delta_{0}$ and $\delta_{1}$ are elementary ranks, i.e., $\mathrm{V}_{\delta_{0}} \prec \mathrm{V}_{\delta_{1}} \prec \mathrm{V}$. In that case, it is possible to iterate the forcings to make $\mathrm{MP}_{<\kappa_{0}-\text { dir. cl. }}\left(H_{\kappa_{0}+}\right)$ and $\mathrm{MP}_{<\kappa_{1}-\text { dir. cl. }}\left(H_{\kappa_{1}+}\right)$ true.

Indeed, let $G$ be $\operatorname{Col}\left(\kappa_{0},<\delta_{0}\right)$-generic over $\mathrm{V}$. Then by Theorem 2.10, $\mathrm{MP}_{<\kappa_{0}-\text { dir. cl. }}\left(H_{\kappa_{0}+}\right)$ holds in $\mathrm{V}[G]$. But obviously, $\left(\mathrm{V}_{\delta_{1}}\right)^{\mathrm{V}[G]} \prec \mathrm{V}[G], \kappa_{1}$ is regular and $\delta_{1}$ is inaccessible in $\mathrm{V}[G]$. So, letting $H$ be $\left.\mathbb{Q}=\left(\operatorname{Col}\left(\kappa_{1},<\delta_{1}\right)\right)^{\mathrm{V}[G]}\right]_{-}$ generic over $\mathrm{V}[G]$, the same theorem gives that $\mathrm{MP}_{<\kappa_{1}-\text { dir. cl. }}\left(H_{\kappa_{1}+}\right)$ holds in $\mathrm{V}[G][H]$. But by Lemma 4.2 , since $\mathbb{Q}$ is $<\kappa_{1}$-directed-closed in $\mathrm{V}[G]$ (and $\kappa_{1} \geq \kappa_{0}^{+}$in $\left.\mathrm{V}[G]\right)$, it follows that $\mathrm{MP}_{<\kappa_{0}-\text { dir. cl. }}\left(H_{\kappa_{0}+}\right)$ is carried over from $\mathrm{V}[G]$ to $\mathrm{V}[G][H]$.

This shows one direction of the following observation:

Observation 5.1 The theory 8

- ZFC,

- $\kappa_{0}$ and $\kappa_{1}$ are regular,

${ }^{8}$ More formally, the theory has to be formulated in a language with additional constant symbols to be interpreted by $\kappa_{0}, \kappa_{1}, \delta_{0}$ and $\delta_{1}$. I omit this formalism because I'm only interested in models where these constants are interpreted in the same way, and moreover, I have been rather formal about these issues earlier on, so that it should be clear by now what is meant. 
- $\delta_{0}=\kappa_{0}^{+}, \delta_{1}=\kappa_{1}^{+}$and $\delta_{0} \leq \kappa_{1}$,

- $\mathrm{MP}_{<\kappa_{0}-\text { dir. cl. }}\left(H_{\kappa_{0}+}\right)+\mathrm{MP}_{<\kappa_{1}-\text { dir. cl. }}\left(H_{\kappa_{1}+}\right)$

is transitive model equiconsistent to the theory expressing

- ZFC,

- $\kappa_{0}, \kappa_{1}, \delta_{0}$ and $\delta_{1}$ are regular,

- $\kappa_{0}<\delta_{0} \leq \kappa_{1}<\delta_{1}$,

- $\mathrm{V}_{\delta_{0}} \prec \mathrm{V}$ and $\mathrm{V}_{\delta_{1}} \prec \mathrm{V}$,

locally in $\kappa_{0}, \kappa_{1}, \delta_{0}$ and $\delta_{1}$.

The converse follows from Theorem 3.8. By the way, everything works for $\mathrm{MP}_{<\kappa_{0}-\text { closed }}\left(H_{\kappa_{0}} \cup\left\{\kappa_{0}\right\}\right)$ and $\mathrm{MP}_{<\kappa_{1}-\text { closed }}\left(H_{\kappa_{1}} \cup\left\{\kappa_{1}\right\}\right)$ just as well.

The analogous construction can be carried out in order to obtain models of the lightface versions $\mathrm{MP}_{<\kappa_{0}-\text { dir. cl. }}\left(H_{\kappa_{0}} \cup\left\{\kappa_{0}\right\}\right)+\mathrm{MP}_{<\kappa_{1}-\text { dir. cl. }}\left(H_{\kappa_{1}} \cup\left\{\kappa_{1}\right\}\right)$. In this case, $\delta_{0}$ and $\delta_{1}$ don't need to be inaccessible, but $\kappa_{1}$ has to be preserved as a cardinal by $\operatorname{Col}\left(\kappa_{0},<\delta_{0}\right)$.

So far, only the possibility of combining the maximality principles for directed closed forcings at several regular cardinals was discussed. So the following question is not far-fetched.

Question 5.2 Is $\mathrm{MP}_{\mathrm{Col}\left(\kappa_{0}\right)}\left(H_{\kappa_{0}} \cup\left\{\kappa_{0}\right\}\right)+\mathrm{MP}_{\mathrm{Col}\left(\kappa_{1}\right)}\left(H_{\kappa_{1}} \cup\left\{\kappa_{1}\right\}\right)$ consistent?

I shall give a partial negative answer in section 6 . This reveals a significant difference between $\mathrm{MP}_{\mathrm{Col}(\kappa)}\left(H_{\kappa} \cup\{\kappa\}\right)$ and the other closed maximality principles. The reason why the above method doesn't work for the classes of collapses is that $\operatorname{Col}\left(\kappa_{1},<\delta_{1}\right)$ is not a member of $\operatorname{Col}\left(\kappa_{0}\right)$, so that it's not clear that this forcing will preserve $\mathrm{MP}_{\mathrm{Col}\left(\kappa_{0}\right)}\left(H_{\kappa_{0}} \cup\left\{\kappa_{0}\right\}\right)$.

Let's now analyze the possibility of having $\mathrm{MP}_{<\kappa-\text { dir. cl. }}\left(H_{\kappa^{+}}\right)$simultaneously at each of the first $\alpha$ regular cardinals $\kappa$. Let $\rho(x)$ be a canonical term such that $y=\rho(x)$ if and only if $x$ is an ordinal and $y$ is the $x^{\text {th }}$ regular cardinal.

Given a class term $S$ in one free variable, let

$$
\bigwedge_{\gamma<\dot{\alpha}} \mathrm{MP}_{<\rho(\gamma)-\text { dir. cl. }}(S(\rho(\gamma)))
$$

be the scheme of $\mathcal{L}_{\dot{\alpha}}$-formulae consisting of the following statements:

$$
\begin{gathered}
\forall \gamma<\dot{\alpha} \forall \vec{a} \quad[(\vec{a} \in S(\rho(\gamma)) \wedge \\
\text { " } \varphi(\vec{a}) \text { is }<\rho(\gamma) \text {-directed-closed-forceably necessary" ) } \\
\quad \Longrightarrow \varphi(\vec{a})],
\end{gathered}
$$

for every $\mathcal{L}$-formula $\varphi(\vec{x})$.

So the principle $\bigwedge_{\gamma<\alpha} \mathrm{MP}_{<\rho(\gamma) \text {-dir. cl. }}\left(H_{\rho(\gamma)^{+}}\right)$expresses that for every $\kappa$ among the $\alpha$ first regular cardinals, $\mathrm{MP}_{<\kappa-\text { dir. cl. }}\left(H_{\kappa} \cup\{\kappa\}\right)$ holds. 
The theory corresponding to $S(\kappa)=H_{\kappa^{+}}$will contain a predicate $A$ of regular reflecting ranks. Note that this can be expressed as the scheme consisting of the sentences

$\forall \delta \quad\left(A(\delta) \longrightarrow\left(\right.\right.$ " $\delta$ is regular" $\left.\left.\wedge \forall \vec{x} \in \mathrm{V}_{\delta} \quad\left(\varphi_{\mathrm{V}_{\delta}}(\vec{x}) \Longleftrightarrow \varphi(\vec{x})\right)\right)\right)$,

for any formula $\varphi$ in the language without the additional predicate symbol $A$. I shall write " $A$ consists of regular reflecting ranks" for this scheme. I shall also say that a class of ordinals is discrete if it contains no limit point of itself.

Theorem 5.3 If $\bigwedge_{\gamma<\alpha} \mathrm{MP}_{<\rho(\gamma)-\text { dir. cl. }}\left(H_{\rho(\gamma)^{+}}\right)$is consistent, then so is the theory, formulated in the language of set theory with an additional 1-ary predicate symbol A, consisting of the following axioms:

- $\mathrm{ZFC}_{A}$,

- A is a discrete class of ordinals, with order-type $\alpha$,

- A consists of regular reflecting ranks.

Again, for all we know yet, $\alpha=\infty$ is possible..$^{9}$

Proof. Assume $\bigwedge_{\gamma<\alpha} \mathrm{MP}_{<\rho(\gamma)-\text { dir. cl. }}\left(H_{\rho(\gamma)+}\right)$. Let $S$ consist of the successors of the first $\alpha$ regular cardinals. Then, by Theorem 3.8, for every $\delta \in S$,

$$
\left\langle L_{\delta}, \in\right\rangle \prec\langle L, \in\rangle,
$$

and $\delta$ is inaccessible in $L$. Moreover, $S$ is discrete, so the natural candidate for a model of $T^{<\alpha}$ would now seem to be $L[S]^{10}$ and one would hope that for every $\delta \in S$, it would be the case that $\left\langle\left|L_{\delta}[S]\right|, \in\right\rangle \prec\langle|L[S]|, \in\rangle$. But note that this would imply that $|L[S]|=L$, and there is no reason why that should be the case. But the latter observation puts us in the right direction for finding the desired model and the desired interpretation of $\dot{A}$.

Namely, set:

$$
A^{\prime}=\left\{\delta \mid \exists \epsilon \in S \quad\left\langle L_{\delta}, \epsilon\right\rangle \prec\left\langle L_{\epsilon}, \epsilon\right\rangle \wedge \delta \text { is inaccessible in } L\right\}
$$

Then, if $\epsilon \in S$ or $\epsilon$ is a limit point of $S$,

$$
A^{\prime} \cap \epsilon=\left\{\delta \mid\left\langle L_{\delta}, \epsilon\right\rangle \prec\left\langle L_{\epsilon}, \in\right\rangle \wedge \delta \text { is inaccessible in } L\right\} \in L,
$$

and so, $L=\left|L\left[A^{\prime}\right]\right|$. Hence, if $A$ consists of the first $\alpha$ elements of $A^{\prime}$ which are no limit points of $A^{\prime},\langle L, \in, A\rangle=L[A]$ is a model of the desired theory. Clearly, it is a model of $\mathrm{ZFC}_{\dot{A}}+$ " $\dot{A}$ has order-type $\alpha$, is discrete, and consists of

\footnotetext{
${ }^{9}$ As before, I am not distinguishing between $\dot{\alpha}$ and its interpretation, since I shall always be interested in models in which the interpretation is the same.

${ }^{10}$ If $M$ is a model, I denote its universe by $|M|$. When treating $L[A]$, I use Jensen's terminology that $L[A]=\langle|L[A]|, \in, A \cap|L[A]|\rangle$.
} 
inaccessible cardinals". And if $\delta \in A$, then letting $\epsilon \in S$ witness that $\delta \in A^{\prime}$, it follows that

$$
\left\langle L_{\delta}, \in\right\rangle \prec\left\langle L_{\epsilon}, \in\right\rangle \prec\langle L, \in\rangle .
$$

Since $\delta$ is inaccessible in $L$, it follows that $L_{\delta}=\left(\mathrm{V}_{\delta}\right)^{L}$, so that in $L$, every instance of the scheme $\left\langle\mathrm{V}_{\delta}, \in\right\rangle \prec\langle L, \in\rangle$ holds.

The converse direction works only if $\alpha<\infty$ :

Theorem 5.4 Assuming the theory of the previous theorem with $\alpha<\infty$, that is, $\mathrm{ZFC}_{A}+\operatorname{otp}(A)=\alpha<\infty+$ " $A$ is discrete and $A$ consists of regular reflecting ranks", there is a forcing extension which satisfies

$$
\mathrm{ZFC}+\bigwedge_{\gamma<\alpha} \mathrm{MP}_{<\rho(\gamma)-\text { dir. cl. }}\left(H_{\rho(\gamma)^{+}}\right) .
$$

Proof. Let $A$ be the discrete set of order-type $\alpha$ which consists of inaccessible cardinals the ranks of which are elementary in V. By the trick that was used in the proof of Theorem 5.3, I may additionally assume $\mathrm{V}=L$, of which I shall only use $\mathrm{GCH}$.

Let $\left\langle\beta_{\gamma} \mid \gamma<\alpha\right\rangle$ enumerate $A$. For $\gamma<\alpha$, let $\bar{\beta}_{\gamma}$ be the least regular cardinal which is larger than or equal to $\sup _{\delta<\gamma} \beta_{\delta}<\beta_{\gamma}$. So $\bar{\beta}_{\gamma}<\beta_{\gamma}$ as $A$ is discrete.

Joel Hamkins likes to describe his method of producing a model of $\operatorname{MP}\left(H_{\omega_{1}}\right)$ as "running through the house and turning on all the lights". I do the same thing, only now the house has a lot of levels. So I start at the ground floor and work my way up through the first $\alpha$ levels.

More precisely, I am going to perform a length $\alpha$ reverse Easton iteration of Lévy-collapses, collapsing $\beta_{\gamma}$ to be $\bar{\beta}_{\gamma}^{+}$for every $\gamma<\alpha$. The iteration has the form $\left\langle\left\langle\mathbb{P}_{\gamma} \mid \gamma \leq \alpha\right\rangle,\left\langle\mathbb{Q}_{\gamma} \mid \gamma<\infty\right\rangle\right\rangle$, where $\mathbb{P}_{\gamma+1} \cong \mathbb{P}_{\gamma} * \mathbb{Q}_{\gamma}$.

Let $\mathbb{P}_{0}=\{\emptyset\}$ be trivial forcing, and if $\mathbb{P}_{\gamma}$ is defined, then let $\mathbb{Q}_{\gamma}$ be a $\mathbb{P}_{\gamma}$-name for $\operatorname{Col}\left(\bar{\beta}_{\gamma},<\beta_{\gamma}\right)$. At limit $\lambda \leq \alpha$, take the limit of the previous construction, with Easton support. More precisely, let $\mathbb{P}_{\lambda}$ consist of those sequences $\vec{p}$ of length $\lambda$ such that for all $\gamma<\lambda, \vec{p} \mid \gamma \in \mathbb{P}_{\gamma}$, and for every regular cardinal $\rho$, the set $\rho \cap\left\{\beta_{\gamma} \mid \gamma \in \operatorname{support}(\vec{p})\right\}$ is bounded in $\rho$.

Now let $G$ be $\mathbb{P}=\mathbb{P}_{\alpha}$-generic over V. For every $\gamma<\alpha, \mathbb{P}$ can be decomposed into a product $\mathbb{P} \cong \mathbb{P}_{\gamma} * \mathbb{Q}_{\gamma} * \mathbb{P}^{\gamma}$, where:

1. $\overline{\overline{\mathbb{P}_{\gamma}}} \leq \bar{\beta}_{\gamma}$ and $\mathbb{P}_{\gamma}$ is $\bar{\beta}_{\gamma}$-c.c.,

2. $\Vdash_{\mathbb{P}_{\gamma}} \mathbb{Q}_{\gamma}=\operatorname{Col}\left(\bar{\beta}_{\gamma},<\beta_{\gamma}\right)$,

3. $\mathbb{P}_{\gamma} * \mathbb{Q}_{\gamma} \Vdash \mathbb{P}^{\gamma}$ is $<\bar{\beta}_{\gamma+1}$-directed-closed.

It is obvious that $\left\{\bar{\beta}_{\gamma} \mid \gamma<\alpha\right\}$ are the first $\alpha$ regular cardinals of $\mathrm{V}[G]$. Now let $\gamma<\alpha$, and set $\kappa=\bar{\beta}_{\gamma}, \delta=\beta_{\gamma}=\bar{\beta}_{\gamma+1}$. Let $G_{\gamma}$ be V-generic for $\mathbb{P}_{\gamma}, H$ be $\mathbb{Q}_{\gamma}^{G_{\gamma}}$-generic over $\mathrm{V}\left[G_{\gamma}\right]$, and $G^{\gamma}$ be $\mathbb{P}^{\gamma} G_{\gamma} * H$-generic over $\mathrm{V}\left[G_{\gamma}\right][H]$, so that $G \cong G_{\gamma} * H * G^{\gamma}$. Since by $1 ., \mathbb{P}_{\gamma}$ is smaller than $\delta$, it follows that $\mathrm{V}_{\delta}^{\mathrm{V}\left[G_{\gamma}\right]} \prec \mathrm{V}\left[G_{\gamma}\right] . \kappa$ is a regular cardinal in $\mathrm{V}\left[G_{\gamma}\right]$, as $\mathbb{P}_{\gamma}$ is $\kappa$-c.c. Over $\mathrm{V}\left[G_{\gamma}\right]$, 
$H$ is $\operatorname{Col}(\kappa,<\delta)$-generic, by 2 . So by Theorem $2.10, \mathrm{~V}\left[G_{\gamma}\right][H]$ is a model of $\mathrm{MP}_{<\kappa \text {-dir. cl. }}\left(H_{\kappa^{+}}\right)$. Finally, $\mathrm{V}\left[G_{\gamma}\right][H]\left[G^{\gamma}\right]$ is a $<\bar{\beta}_{\gamma+1^{-}}$-directed-closed extension of $\mathrm{V}\left[G_{\gamma}\right][H]$ (where $\bar{\beta}_{\gamma+1}$ is still regular, as $\operatorname{Col}(\kappa,<\delta)$ is $\delta$-c.c.). But $\kappa<\delta$, so by Lemma $4.2, \mathrm{MP}_{<\kappa-\text { dir. cl. }}\left(H_{\kappa^{+}}\right)$remains true in $\mathrm{V}\left[G_{\gamma}\right][H]\left[G^{\gamma}\right]=\mathrm{V}[G]$. Since $\gamma<\alpha$ was chosen arbitrarily, this shows that $\bigwedge_{\gamma<\alpha} \mathrm{MP}_{<\rho(\gamma) \text {-dir. cl. }}\left(H_{\rho(\gamma)^{+}}\right)$ holds in $\mathrm{V}[G]$.

But what about the global principle $\bigwedge_{\gamma<\infty} \mathrm{MP}_{<\rho(\gamma)-\text { dir. cl. }}\left(H_{\rho(\gamma)^{+}}\right)$?

Definition 5.5 Let $\varphi$ be a sentence, maybe containing parameters $\vec{a}$.

1. $\varphi$ is local, if there is a cardinal $\lambda$ and a finite subtheory of ZFC such that for any two transitive, set-sized models $M$ and $N$ of that theory, both containing $\vec{a}$, with the same ordinals and with $\left(H_{\lambda}\right)^{M}=\left(H_{\lambda}\right)^{N}$, it follows that $M \models \varphi$ iff $N=\varphi$.

2. Let $\kappa$ be a regular cardinal. Then $\varphi$ is provably $<\kappa$-directed-closedforceably necessary if there is a finite subtheory of ZFC such that $\varphi$ is $<\kappa$-directed-closed-forceably necessary over any set-sized transitive model $M$ of that finite theory, which contains $\kappa$ and $a$.

When trying to force a model of $\bigwedge_{\gamma<\infty} \mathrm{MP}_{<\rho(\gamma) \text {-dir. cl. }}\left(H_{\rho(\gamma)^{+}}\right)$from a model of $T^{<\infty}$ in the "obvious" way, the resulting model falls a bit short.

Theorem 5.6 Assuming $\mathrm{ZFC}_{A}+$ " $A$ is an unbounded, discrete class of regular reflecting ranks", there is a proper class iterated forcing extension $W$ of $\mathrm{V}$, in which for every formula $\varphi$, the following is true: If $\kappa$ is a regular cardinal, $\vec{a} \in$ $H_{\kappa^{+}}$and $\varphi(\vec{a})$ is a local statement that's provably $<\kappa$-directed-closed-forceably necessary, then $\varphi(\vec{a})$ holds.

Proof. Let $\mathbb{P}=\mathbb{P}^{\infty}$ be the result of carrying out the forcing iteration described in the proof of Theorem 5.4, this time with length $\infty$. Let $G$ be $\mathbb{P}$-generic over $\mathrm{V}$, and let $W=\mathrm{V}[G]$. It is well-known that this kind of proper class iteration yields a model of ZFC.

As before, let $\left\langle\beta_{\gamma} \mid \gamma<\infty\right\rangle$ enumerate $A$ (where $A$ is the interpretation of $\dot{A})$, and let $\bar{\beta}_{\gamma}$ be the least regular cardinal $\geq \sup _{\delta<\gamma} \beta_{\delta}$.

By the argument of the above proof, it follows (in the notation of that proof) that

$$
\mathrm{V}\left[G_{\alpha}\right] \models \bigwedge_{\gamma<\alpha} \mathrm{MP}_{<\rho(\gamma)-\text { dir. cl. }}\left(H_{\rho(\gamma)^{+}}\right)
$$

for any $\alpha$.

Now, from $W$ 's point of view, let $\kappa$ be regular, and let $\varphi(\vec{a})$ be a local statement that's provably $<\kappa$-directed-closed-forceably necessary. Let $T$ be a finite fragment of ZFC witnessing both of these properties of $\varphi(\vec{a})$, and let $\lambda$ 
witness that $\varphi(\vec{a})$ is local. $\lambda$ may be assumed at least as large as $\kappa$ (see the definition).

Fix some $\alpha$ which is large enough that $\bar{\beta}_{\alpha} \geq \lambda, \kappa^{+}$and $\vec{a} \in \mathrm{V}\left[G_{\alpha}\right]$. So $\kappa$ is less than the $\alpha^{\text {th }}$ regular cardinal of $\mathrm{V}[G]$. Since $\mathrm{V}[G]$ is a $<\bar{\beta}_{\alpha+1}$-directed-closed extension of $\mathrm{V}\left[G_{\alpha}\right]$, it follows that

$$
H_{\lambda}^{\mathrm{V}\left[G_{\alpha}\right]}=H_{\lambda}^{\mathrm{V}[G]} .
$$

Now pick some $\gamma$ with the property that $M:=\mathrm{V}_{\gamma}^{\mathrm{V}\left[G_{\alpha}\right]}$ and $N:=\mathrm{V}_{\gamma}^{V[G]}$ are models of $T, H_{\lambda}^{M}=H_{\lambda}^{N}$, and $\vec{a} \in M, N$. This is possible by a straightforward application of Levy's reflection theorem: The class of $\gamma$ which reflect enough properties of $\mathrm{V}[G]$ and $\mathrm{V}\left[G_{\alpha}\right]$ contains a club. In addition, one can ensure that $\gamma$ is $\Sigma_{n}$-reflecting both in $\mathrm{V}[G]$ and in $\mathrm{V}\left[G_{\alpha}\right]$, where both $\varphi(\vec{x})$ and the statement that $\varphi(\vec{x})$ is $<\kappa$-directed-closed-forceably necessary are $\Sigma_{n}$.

Then $\varphi(\vec{a})$ is $<\kappa$-directed-closed-forceably necessary over $\mathrm{V}\left[G_{\alpha}\right]$, as this is true in $M$, because $M$ models $T$, and hence true in $\mathrm{V}\left[G_{\alpha}\right]$, by reflection. So by (1), $\varphi(\vec{a})$ is true in $\mathrm{V}\left[G_{\alpha}\right]$. By $\Sigma_{n}$-elementarity again, $\varphi(\vec{a})$ holds in $M$. Since $\varphi(\vec{a})$ is local, from $W$ 's point of view, it follows that $\varphi(\vec{a})$ holds in $N$ as well. And since $N$ is $\Sigma_{n}$-elementary in $\mathrm{V}[G], \varphi[\vec{a}]$ holds in $\mathrm{V}[G]$.

\section{Remark 5.7}

1. In view of the previous theorem, it's worthwhile to note that all the consequences of the closed Maximality Principles stated in Theorems 3.1, 3.3, 3.6, 3.7 and 3.8 are local with respect to some finite fragment of ZFC, and of course they are provably forceably necessary. This means in particular, that if the theory in the previous theorem holds (i.e., the scheme of formulae expressing that for every formula $\varphi(\vec{x})$, if $\kappa$ is regular, $\vec{a} \in H_{\kappa^{+}}, \varphi(\vec{a})$ is local and provably $<\kappa$-directed-closed-forceably necessary, then $\varphi(\vec{a})$ is true), then the theory $\mathrm{ZFC}_{A}+$ " $A$ is a discrete proper class consisting of regular reflecting ranks" holds in an inner model (because every instance of the scheme $L_{\kappa^{+}} \prec L$ follows; the argument of Theorem 5.3 goes thru). So these theories are equiconsistent.

2. Also, it's quite obvious that the construction in the previous proof shows that one can produce a model of the above scheme, where $H_{\kappa^{+}}$is replaced with $H_{\kappa}$, just assuming the consistency of ZFC. In such a model, the statements concerning the existence of trees which are able to realize equivalence relations, which I mentioned in the section on the automorphism tower problem (see Theorem 3.15), are true, since they are local. So this yields a "canonical theory" which implies the statements shown consistent in [HT00].

Another such theory, which at least implies the existence of trees which are able to realize bounded equivalence relations, and hence the existence 
of groups with highly malleable automorphism towers, is $\mathrm{V}=L$, as was shown in [FHOY].

The reason why it was not possible to prove the full principle $\bigwedge_{\gamma<\infty} \mathrm{MP}_{<\rho(\gamma) \text {-dir. cl. }}\left(H_{\rho(\gamma)^{+}}\right)$using the previous proof, was that the final model $\mathrm{V}[G]$ is a proper class forcing extension of $\mathrm{V}\left[G_{\beta}\right]$, for any $\beta$. I shall show in the following section that there is no way to overcome this problem...

But before doing that, I want to take a look at combining the very lightface principles $\mathrm{MP}_{<\kappa-\text { dir. cl. }}(\emptyset)$ for various $\kappa$.

Lemma 5.8 If ZFC is consistent, then so is the theory

$$
\mathrm{ZFC}+\bigwedge_{\gamma<\infty} \mathrm{MP}_{<\rho(\gamma)-\text { dir. cl. }}(\emptyset) .
$$

Proof. Let's work in a universe in which there is a proper class $A$ consisting of ordinals $\delta$ such that $\mathrm{V}_{\delta} \prec \mathrm{V}$, and let $\Omega \in A$ be very closed and have large cofinality. Using the usual arguments involving compactness and Levy's reflection theorem, it is easy to see that the consistency strength of this is the same as that of ZFC.

I shall construct a forcing iteration $\left\langle\left\langle\mathbb{P}_{\gamma}, \mathbb{Q}_{\gamma}\right\rangle \mid \gamma<\theta\right\rangle$, where $\theta$ is countable, as follows:

$\mathbb{P}_{0}$ is trivial forcing. Let $\kappa_{0}$ be the least regular cardinal such that there is a formula $\varphi_{0}$ such that $\mathrm{V}_{\Omega}$ thinks that $\varphi_{0}$ is $<\kappa_{0}$-directed-closed-forceably necessary but not $<\kappa_{0}$-directed-closed-necessary (if there is such a regular cardinal. Otherwise, don't force at all.). In other words, $\kappa_{0}$ is least such that

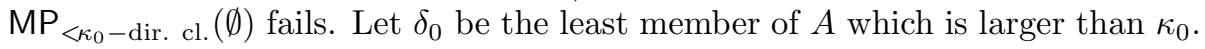
Then let $\mathbb{Q}_{0}$ be (a $\mathbb{P}_{0}$-name for) $\operatorname{Col}\left(\kappa_{0},<\delta_{0}\right)$.

At limit $\lambda$, take the limit of the previous construction, with full support.

If $\mathbb{P}_{\alpha}$ is defined, then let $\kappa_{\alpha}$ be the least regular cardinal in $\mathrm{V}_{\Omega}^{\mathbb{P}_{\alpha}}$ such that there is a formula $\varphi_{\alpha}$ such that $\mathrm{V}_{\Omega}^{\mathbb{P}_{\alpha}}$ thinks that $\varphi$ is $<\kappa_{\alpha}$-directed-closedforceably necessary but not $<\kappa_{\alpha}$-directed-closed-necessary, if such a thing exists, otherwise $\theta=\alpha$ and the iteration stops. Again, this is the same as to say that

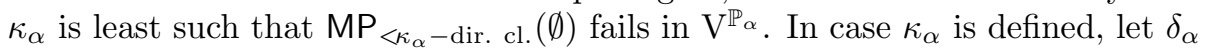
be the least member of $A$ which is above $\kappa_{\alpha}$ and greater than the cardinality of $\mathbb{P}_{\alpha}$, and let $\mathbb{Q}_{\alpha}$ be a $\mathbb{P}_{\alpha}$-name for $\operatorname{Col}\left(\kappa_{\alpha},<\delta_{\alpha}\right)$.

It is easy to see that the $\kappa_{\alpha}$ 's are strictly increasing, using Observation 4.1 and Theorem 2.8. Further, the map $\alpha \mapsto \varphi_{\alpha}$ is injective, because at stage $\alpha$ of the iteration, $\varphi_{\alpha}$ has become (necessarily) $<\kappa_{\alpha}$-directed-closed-necessary and the tail forcing after stage $\alpha$ is $<\kappa_{\alpha}$-directed-closed. So $\varphi_{\alpha}$ can never again witness the existence of some formula which is not $<\kappa$-directed-closed-necessary, for some larger $\kappa$. Since there are only countably many formulae, the iteration must terminate at some countable stage.

By the same argument, one can see that one can have the maximality principles for directed closed forcings simultaneously at all regular cardinals, as long as the parameters which are allowed in the formulae altogether form a set. In the next section, the reason for the necessity of this restriction will become clear. 


\section{Maximality principles and the Ground Axiom}

The following axiom was introduced by Hamkins and Reitz.

Definition 6.1 The Ground Axiom GA says that V is not a nontrivial setforcing extension of an inner model.

In [Rei06], it is shown that the statement "V is a forcing extension of an inner model by the forcing notion $\mathbb{P}$ " is expressible by a first order formula which I shall denote by $\Psi(\mathbb{P}) .{ }^{11}$ So $G A$ says: $\forall \mathbb{P} \quad(\mathbb{P}$ is non-atomic $\Longrightarrow \neg \Psi(\mathbb{P}))$. Note that $\Psi$ does not depend on $\mathrm{V}$; it is uniform.

This limits the possible ways of producing models of the maximality principles under investigation.

Lemma 6.2 Any of the maximality principles introduced imply the failure of $\mathrm{GA}$. More specifically, $\mathrm{MP}_{<\kappa-\text { closed }}\left(H_{\kappa} \cup\{\kappa\}\right)$ implies that there is a nontrivial $<\kappa$-closed $\mathbb{P}$ such that $\Psi(\mathbb{P})$ holds, $\mathrm{MP}_{<\kappa-\text { dir. cl. }}\left(H_{\kappa} \cup\{\kappa\}\right)$ implies that there is such a $\mathbb{P}$ that's $<\kappa$-directed-closed, and $\mathrm{MP}_{\mathrm{Col}(\kappa)}\left(H_{\kappa} \cup\{\kappa\}\right)$ implies that there is such a $\mathbb{P}$ of the form $\operatorname{Col}(\kappa,<\delta)$.

Proof. The statement that there is a $\mathbb{P}$ as in the statement of the lemma is obviously forceably necessary in the appropriate sense. So it is true, by the appropriate maximality principle.

In order to conclude more, it is necessary to give some background on the $\delta$-approximation and -cover properties introduced by Hamkins in [Ham03a].

Definition 6.3 (Hamkins) Let $W \subseteq V$ be transitive models of ZFC $_{\delta}$, where $\delta$ is regular in $V .^{12}$

1. $W$ has the $\delta$-cover property with respect to $V$ if for every $A \subseteq W, A \in V$, such that $\overline{\bar{A}}^{V}<\delta$, there is a $B \in W$ such that $A \subseteq B$ and $\overline{\bar{B}}^{W}<\delta$.

2. For $A \subseteq W, A \in V$, a $\delta$-approximation to $A$ over $W$ is a set of the form $A \cap B$, where $B \in W$ and $\overline{\bar{B}}^{W}<\delta$. W has the $\delta$-approximation property with respect to $V$ if whenever $A \subseteq W$ with $A \in \mathrm{V}$ is such that every $\delta$-approximation to $A$ over $W$ is an element of $W$, it follows that $A \in W$.

The following facts will be needed:

\footnotetext{
${ }^{11} \Psi(\mathbb{P})=\exists \delta \exists z \exists G \quad \Phi(\delta, z, \mathbb{P}, G)$, where $\Phi$ is the formula introduced in [Rei06, Section 2].

${ }^{12} \mathrm{ZFC}_{\delta}$ is the fragment of ZFC in which replacement is only postulated for definable functions with domain $\leq \delta$, and the statement that every set is coded by a set of ordinals, in the following sense:

$$
\forall x \exists \alpha \exists e \subseteq \alpha \times \alpha \quad\langle\operatorname{TC}(\{x\}), \in\rangle \cong\langle\alpha, e\rangle .
$$
}


Lemma 6.4 (Hamkins, Laver) If $M, M^{\prime} \subseteq V$ are transitive models of $\mathrm{ZFC}_{\delta}$ with the same ordinals, where $\delta$ is regular in $V$, both $M$ and $M^{\prime}$ satisfy the $\delta$-approximation and-cover properties with respect to $V,\left(\delta^{+}\right)^{M}=\left(\delta^{+}\right)^{M^{\prime}}=$ $\left(\delta^{+}\right)^{V}$ and $\mathcal{P}(\delta) \cap M=\mathcal{P}(\delta) \cap M^{\prime}$, then $M=M^{\prime}$.

A proof of this, which is due to Hamkins, can be found in [Rei06, Lemma 7.2]. The following definition facilitates the statement of the next lemma:

Definition 6.5 (Hamkins) A forcing notion has a closure point at a cardinal $\delta$ if it factors as $\mathbb{P} * \dot{\mathbb{Q}}$, where $\overline{\overline{\mathbb{P}}} \leq \delta, \mathbb{P}$ is nontrivial, and $\Vdash_{\mathbb{P}} \dot{\mathbb{Q}}$ is $<\delta^{+}$-strategically closed.

It has a strong closure point at $\delta$ if it factors as $\mathbb{P} * \dot{\mathbb{Q}}$ if, where $\mathbb{P}$ is as above, and $\mathbb{Q}$ is $<\delta^{++}$-strategically closed.

The following lemma will be crucial.

Lemma 6.6 ([Ham03a, Lemma 13]) Let $M \subseteq V$ be models of ZFC. Let $G$ be generic over $M$ for a partial order $\mathbb{P} \in M$ so that $V=M[G]$ and $\delta$ is a closure point of $\mathbb{P}$. Then $M$ has the $\left(\delta^{+}\right)^{M}=\left(\delta^{+}\right)^{V}$-approximation and -cover properties with respect to $V$.

Putting the previous two lemmas together results in:

Lemma 6.7 Let $M \subseteq \mathrm{V}$ be models of ZFC. Let $G$ be generic over $M$ for a partial order $\mathbb{P} \in M$ so that $\mathrm{V}=M[G]$ and $\delta$ is a strong closure point of $\mathbb{P}$. Then the following is true in $\mathrm{V}$ :

$$
M=\bigcup_{\alpha \in B \backslash \gamma} X_{\alpha}
$$

where $B$ is the set of fixed points of the $\beth$-function with cofinality $>\delta^{+}, X_{\alpha}$ is the unique transitive model $N$ with:

- $N \models \mathrm{ZFC}_{\delta^{+}}$,

- On $\cap N=\alpha$,

- $\left(\delta^{++}\right)^{N}=\left(\delta^{++}\right)^{\mathrm{V}}$,

- $N$ has the $\delta^{+}$-approximation and-cover properties with respect to $V_{\alpha}$,

- $\mathcal{P}\left(\delta^{+}\right) \cap N=z$, where $z=\mathcal{P}\left(\delta^{+}\right) \cap M$,

if such $N$ exists, and $\gamma$ is least such that for any $\beta>\alpha>\gamma$ with $\alpha, \beta \in B, X_{\alpha}$ and $X_{\beta}$ exist and $X_{\alpha} \subseteq X_{\beta}$.

So $M=\{x \mid \mathrm{V} \models \psi(x, z)\}$, for some first order formula $\psi$. 
Proof. The point here is that for $\overline{\overline{\mathbb{P}}} \cup \delta^{++}<<\alpha \in B, \mathrm{~V}_{\alpha}^{M}$ is the unique model with the properties listed in the lemma. To see this, it suffices to check that $\mathrm{V}_{\alpha}^{M}$ does satisfy these requirements, since uniqueness then follows from Lemma 6.4. So let's go through the list: $\alpha$ is a fixed-point of the $\beth$-function, as computed in $M$ as well, and of course the cofinality of such $\alpha$ is also from $M$ 's point of view larger than $\delta^{+}$. So $\mathrm{V}_{\alpha}^{M}$ is a model of $\mathrm{ZFC}_{\delta^{+}}$(for the verification of this fact, I refer the reader to [Rei06]). That $\mathrm{V}_{\alpha}^{M}$ and $\mathrm{V}_{\alpha}$ have the same ordinals is trivial. Since $\delta$ is a strong closure point of $\mathbb{P}$, it follows that $M$ and $\mathrm{V}$ agree about the value of $\delta^{++}$, hence so do $\mathrm{V}_{\alpha}^{M}$ and $\mathrm{V}_{\alpha}$. By Lemma 6.6, $M$ has the $\delta^{+}$-approximation and -cover properties with respect to $\mathrm{V}$, and it follows immediately that $\mathrm{V}_{\alpha}^{M}$ has these properties with respect to $\mathrm{V}_{\alpha}$. And of course, $\mathrm{V}_{\alpha}^{M}$ has the same subsets of $\delta^{+}$that $M$ has.

Lemma 6.8 Assume that $\mathrm{MP}_{<\kappa-\operatorname{closed}}(S)$ holds. Then the universe is not a forcing extension of an inner model by a forcing notion $\mathbb{P}$ of cardinality $\delta$ such that

1. $\delta^{+}<\kappa$,

2. $\mathcal{P}\left(\mathcal{P}\left(\delta^{+}\right)\right) \subseteq S$ and $\kappa \in S$.

Proof. Assume the contrary, so that the universe is a forcing extension of an inner model $M$ by a forcing $\mathbb{P}$ as above. Let $z=\mathcal{P}\left(\delta^{+}\right) \cap M$, where $\delta=\overline{\overline{\mathbb{P}}}$. Then $z \in S$, by assumption.

The point is now that if $\dot{\mathbb{Q}}$ is any $\mathbb{P}$-name for a (possibly trivial) forcing such that $\Vdash_{\mathbb{P}} \dot{\mathbb{Q}}$ is $<\kappa$-strategically closed, then $\delta$ is a strong closure point of the composed forcing $\mathbb{P} * \dot{\mathbb{Q}}$, so that by Lemma $6.7, M$ is uniformly definable in the corresponding extension, using $z$ as a parameter.

So in any such extension $N$ of $M$, it will be the case that $M=\{x \mid \psi(x, z)\}^{N}$, where $\psi$ is the formula from Lemma 6.7. In favor of readability, let's write $M$ for this term defining $M$ in any extension of $M$ like $N$.

Then the statement " $\left(\kappa^{+}\right)^{M}<\kappa^{+}$" is clearly $<\kappa$-closed-forceably necessary over $\mathrm{V}$, and hence true in $\mathrm{V}$ - note that the only parameters occurring in that statement are $\kappa$ and $z$, both of which are in $S$. So this is already true in V. But this cannot be the case, since $\mathrm{V}$ is a forcing extension of $M$ by a forcing of size $<\kappa$. This is a contradiction.

Theorem 6.9 The theory ZFC $+\bigwedge_{\gamma<\infty} \mathrm{MP}_{<\rho(\gamma)-\operatorname{closed}}\left(H_{\rho(\gamma)} \cup\{\rho(\gamma)\}\right)$ is inconsistent.

Proof. It is clear by now that the above theory implies that the ground axiom fails. So working in a model of that theory, let $\mathbb{P}$ be such that $\mathrm{V}=M[G]$, for some partial order, some inner model $M$ and some generic $G$. Let $\delta=\overline{\overline{\mathbb{P}}}$, and pick a regular $\kappa>\left(2^{\delta^{+}}\right)$. Then $\mathrm{MP}_{<\kappa-\text { closed }}\left(H_{\kappa} \cup\{\kappa\}\right)$ holds by assumption. But $\mathcal{P}\left(\mathcal{P}\left(\delta^{+}\right)\right) \subseteq H_{\kappa}$, because every subset of $\mathcal{P}\left(\delta^{+}\right)$has hereditary cardinality 
$\leq 2^{\delta^{+}}$. This contradicts Lemma 6.8 .

Clearly, the previous argument establishes that it cannot be the case that every set $x$ is in the parameter set for which $\mathrm{MP}_{<\kappa-\text { closed }}$ holds, for unboundedly many regular $\kappa$.

Corollary 6.10 $\mathrm{MP}_{<\kappa-\operatorname{closed}}\left(H_{\kappa^{+}}\right)$implies that the universe is not a forcing extension of an inner model by a nontrivial forcing $\mathbb{P}$ such that $\overline{\overline{\mathbb{P}}}^{+}<\kappa$.

Proof. Let $\delta=\overline{\overline{\mathbb{P}}}$. Note that by Lemma 3.1, it follows that $2^{<\kappa}=\kappa$, as $\mathrm{MP}_{<\kappa-\text { closed }}\left(H_{\kappa} \cup\{\kappa\}\right)$ holds. This means that $\mathcal{P}\left(\mathcal{P}\left(\delta^{+}\right)\right) \subseteq H_{\kappa^{+}}$, since every subset of $\mathcal{P}\left(\delta^{+}\right)$has hereditary cardinality $\leq 2^{\delta^{+}} \leq \kappa$. Again, this contradicts Lemma 6.8 .

I will close the paper by giving a partial answer to Question 5.2.

Lemma 6.11 If $\mathrm{MP}_{\mathrm{Col}\left(\kappa_{0}\right)}\left(H_{\kappa_{0}} \cup\left\{\kappa_{0}\right\}\right)$ holds, then there can be no $\kappa_{1}>\kappa_{0}^{+}$ such that $\mathrm{MP}_{<\kappa_{1}-\text { closed }}\left(H_{\kappa_{1}+}\right)$ holds.

Proof. The point is that $\mathrm{MP}_{\mathrm{Col}\left(\kappa_{0}\right)}\left(H_{\kappa_{0}} \cup\left\{\kappa_{0}\right\}\right)$ implies that the universe is a forcing extension of an inner model $M$ by a forcing $\mathbb{P}$ of cardinality $\kappa_{0}$.

Indeed, it is easily seen that the statement "V is a forcing extension of an inner model by $\operatorname{Add}\left(\kappa_{0}\right)$, the forcing to add a Cohen subset of $\kappa_{0}$ " is $\operatorname{Col}\left(\kappa_{0}\right)$ forceably necessary. It is forceable because $\operatorname{Col}\left(\kappa_{0}, \lambda\right)$ is forcing equivalent to $\operatorname{Add}\left(\kappa_{0}\right) \times \operatorname{Col}\left(\kappa_{0}, \lambda\right)$, for suitable $\lambda$. But once true, it is necessary: If $\mathrm{V}=M[G]$, where $G$ is $\operatorname{Add}\left(\kappa_{0}\right)$-generic over $\mathrm{V}$, and $H$ is generic for some $\mathbb{Q} \in \operatorname{Col}\left(\kappa_{0}\right)^{\mathrm{V}}$, it follows that $\mathbb{Q} \in \mathrm{V}$. And so, $N=M[G][H]=M[H][G]$, which means that $N$ is again an $\operatorname{Add}\left(\kappa_{0}\right)$-generic extension of an inner model.

This contradicts the previous corollary.

\section{References}

[AH01] Arthur W. Apter and Joel David Hamkins. Indestructible weakly compact cardinals and the necessity of supercompactness for certain proof schemata. Mathematical Logic Quarterly, 47(4):563-571, 2001.

[Cha00] Christophe Chalons. Full set theory. Electronic Preprint, 2000.

[DJ74] Keith J. Devlin and Håvard Johnsbråten. The Souslin Problem. Lecture Notes in Mathematics 405. Springer, Berlin, 1974.

[FH06] Gunter Fuchs and Joel David Hamkins. Degrees of rigidity for Souslin trees. ArXiv Mathematics eprints, Feb. 2006. 33 pages, Submitted to the Journal of Symbolic Logic. 
[FH07] Gunter Fuchs and Joel David Hamkins. Changing the heights of automorphism towers by forcing with Souslin trees over L. ArXiv Mathematics eprints, Feb. 2007. 23 pages. Submitted to the Journal of Symbolic Logic.

[FK03] Matthew Foreman and Peter Komjath. The club guessing ideal (remarks on a paper by Gitik and Shelah). 2003.

[Ham03a] Joel David Hamkins. Extensions with the approximation and cover properties have no new large cardinals. Fundamenta Mathematicae, 180(3):257-277, 2003.

[Ham03b] Joel David Hamkins. A simple maximality principle. Journal of Symbolic Logic, 68(2):527-550, June 2003.

[HT00] Joel David Hamkins and Simon Thomas. Changing the heights of automorphism towers. Ann. Pure Appl. Logic, 102(1-2):139-157, 2000.

[HW05] Joel David Hamkins and W. Hugh Woodin. The necessary maximality principle for c.c.c. forcing is equiconsistent with a weakly compact cardinal. Mathematical Logic Quarterly, 51(5):493-498, 2005.

[Jec03] Thomas Jech. Set Theory. The Third Millenium Edition. Springer, Berlin, 2003.

[JSSS07] Ronald B. Jensen, Ernest Schimmerling, Ralf Schindler, and John R. Steel. Stacking mice. In preparation, 2007.

[Kun80] Kenneth Kunen. Set Theory. An Introduction To Independence Proofs. North Holland, 1980.

[KY06] Bernhard König and Yasuo Yoshinobu. Kurepatrees and Namba forcing. ArXiv Mathematics eprints, http://arXiv.org/pdf/math/0605130, May 2006.

[Lav78] Richard Laver. Making the supercompactness of $\kappa$ indestructible under $\kappa$-directed closed forcing. Israel Journal of Mathematics, 29(4):385-388, 1978.

[Lei04] George Leibman. Consistency Strengths of Modified Maximality Principles. PhD thesis, The City University of New York, 2004.

[Rei06] Jonas Reitz. The Ground Axiom. PhD thesis, 2006. CUNY Graduate Center.

[SS99] Ernest Schimmerling and John R. Steel. The maximality of the core model. Transactions of the American Mathematical Society, 351(8):3119-3141, March 1999. 
[SV01] Jonathan Stavi and Jouko Väänänen. Reflection principles for the continuum. Logic and Algebra, AMS Contemporary Mathematics Series, 302:59-84, 2001.

[Tho] Simon Thomas. The Automorphism Tower Problem. To appear. 